\title{
A non-renormalization theorem for chiral primary 3-point functions
}

\author{
Marco Baggio, ${ }^{a}$ Jan de Boer $^{a}$ and Kyriakos Papadodimas ${ }^{b}$ \\ ${ }^{a}$ Institute for Theoretical Physics, University of Amsterdam, \\ Science Park 904, Postbus 94485, 1090 GL Amsterdam, The Netherlands \\ ${ }^{b}$ Theory Group, Physics Department, CERN, \\ CH-1211 Geneva 23, Switzerland \\ E-mail: m.baggio@uva.nl, j.deboer@uva.nl, Kyriakos.Papadodimas@cern.ch
}

ABSTRACT: In this note we prove a non-renormalization theorem for the 3-point functions of $1 / 2$ BPS primaries in the four-dimensional $\mathcal{N}=4 \mathrm{SYM}$ and chiral primaries in two dimensional $\mathcal{N}=(4,4)$ SCFTs. Our proof is rather elementary: it is based on Ward identities and the structure of the short multiplets of the superconformal algebra and it does not rely on superspace techniques. We also discuss some possible generalizations to less supersymmetric multiplets.

KEYWORDS: Supersymmetric gauge theory, Gauge-gravity correspondence, AdS-CFT Correspondence

ARXIV EPRINT: 1203.1036 


\section{Contents}

1 Introduction $\quad 2$

2 Background material $\quad 3$

2.1 Conformal perturbation theory 3

2.2 Superconformal Ward identities 4

2.3 Chiral primary 3-point functions 6

2.4 Null vectors in short multiplets 6

$\begin{array}{lll}2.5 & \text { Supersymmetric marginal deformations } & 7\end{array}$

3 Outline of the proof $\quad 8$

4 Two-dimensional CFTs with $\mathcal{N}=(4,4)$ supersymmetry 9

4.1 Short representations and their 3-point functions 9

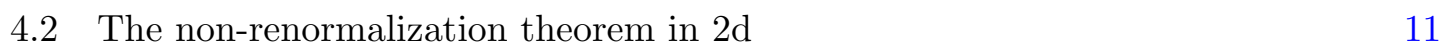

$5 \quad$ Four-dimensional $\mathcal{N}=4$ SCFTs 12

$\begin{array}{lll}5.1 & \text { Short representations } & 13\end{array}$

$\begin{array}{lll}5.2 & \text { The non-renormalization theorem in } 4 \mathrm{~d} & 14\end{array}$

$\begin{array}{llr}6 & \text { Extremal correlators } & 16\end{array}$

$\begin{array}{lll}7 & \text { Other extensions } & 17\end{array}$

$\begin{array}{ll}7.1 \text { Half-chiral states in } 2 \mathrm{~d} \mathcal{N}=(4,4) & 17\end{array}$

$\begin{array}{ll}7.23 \text {-point functions in } 2 \mathrm{~d} \mathcal{N}=(0,4) \text { SCFTs } & 17\end{array}$

$\begin{array}{lll}7.3 & \text { Less supersymmetric multiplets in } 4 \mathrm{~d} & 17\end{array}$

$\begin{array}{lll}8 & \text { Discussions } & 18\end{array}$

$\begin{array}{ll}\text { A Roots and weights } & 19\end{array}$

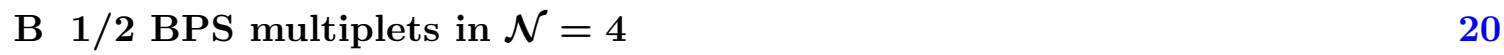

B.1 The $[0, k, 0]$ multiplet $\quad 22$

B.2 The $[0,2,0]$ multiplet 23

C Null states and short multiplets $\quad \mathbf{2 4}$

C.1 Structure of null conditions in $\mathcal{N}=(4,4) \quad 24$

$\begin{array}{ll}\text { C.2 Structure of null conditions for } \mathcal{N}=4 & 25\end{array}$ 


\section{Introduction}

One of the earliest checks of the AdS/CFT correspondence [1-3] was the matching of 3point functions of chiral primaries. This was first done [4-6] for the duality between the $\mathcal{N}=4 \mathrm{SYM}$ and IIB string theory in $\mathrm{AdS}_{5} \times \mathrm{S}^{5}$ and later [7-10] for the duality between the two dimensional $\mathcal{N}=(4,4)$ D1/D5 CFT and IIB string theory on $\mathrm{AdS}_{3} \times \mathrm{S}^{3} \times \mathcal{M}_{4}$. The matching of 3-point functions is non-trivial because they are not fully determined by symmetry considerations.

Notice that a priori the matching did not have to work - i.e. even if it did not work, it would not indicate a problem with the AdS/CFT correspondence. The bulk and boundary computations of 3-point functions are performed at different points of the moduli space (i.e. different values of the coupling constants). In general there is no reason to expect that such computations should give the same answer. The fact that the computations did indeed agree, strongly suggests that these 3-point functions are actually independent of the coupling constant. In other words, that there should exist a "non-renormalization theorem" for 3-point functions of chiral primaries in superconformal field theories with sufficient amount of supersymmetry.

For the case of $\mathrm{AdS}_{5} / \mathrm{CFT}_{4}$ and the $\mathcal{N}=4 \mathrm{SYM}$ a proof of such a non-renormalization theorem was given in a series of works [11-16]. The proof relies on the formalism of analytic superspace. It would be interesting if a more basic proof could be found, which would not depend on the somewhat elaborate construction of analytic superspace. ${ }^{1}$

In the case of $\mathrm{AdS}_{3} / \mathrm{CFT}_{2}$ with $\mathcal{N}=(4,4)$ supersymmetry a (partial) nonrenormalization theorem was proven in [18] using elementary techniques. This theorem is partial because it does not include the most general case of 3-point function of chiral primaries, but only the case of "extremal" correlation functions.

The goal of this work is two-fold. First we complete the non-renormalization theorem of [18] to include the most general 3-point function of chiral primaries in two-dimensional $\mathcal{N}=(4,4)$ theories. $^{2}$ Second, we give a short - and in our view simpler - proof of the non-renormalization theorem for $1 / 2$ BPS chiral primary 3-point functions for $\mathcal{N}=4$ SCFTs in four dimensions. Our presentation provides a unified treatment of both cases, based on superconformal Ward identities and the structure of the representations of the superconformal algebra.

We also prove a few more results:

i) 3-point functions of half-chiral primary states in $2 \mathrm{~d} \mathcal{N}=(4,4)$ SCFTs are not renormalized

ii) 3-point functions of chiral primaries in $2 \mathrm{~d} \mathcal{N}=(0,4)$ SCFTs are not renormalized.

\footnotetext{
${ }^{1}$ In [17] such a proof was proposed. However we believe that the arguments in that paper are actually not sufficient in order to prove the non-renormalization theorem. More explanations about this can be found at the end of section 5.1, in particular see footnote 11.

${ }^{2}$ In this paper we call "chiral primaries" all the operators belonging to the same SU(2) R-symmetry multiplet, and not just the highest-weight state.
} 
iii) "Extremal" $n$-point functions of $1 / 2$ BPS operators in $4 \mathrm{~d} \mathcal{N}=4$ SCFTs are not renormalized

iv) 3-point functions involving one $1 / 4 \mathrm{BPS}$ and two $1 / 2$ BPS operators in $4 \mathrm{~d} \mathcal{N}=4$ SCFTs are not renormalized.

Notice that our results are non-perturbative in the coupling constant of the theory and hold for any gauge group - in particular they do not depend on a large $N$ limit.

The plan of the paper is as follows: in section 2 we present some necessary background material mostly on marginal deformations of CFTs, Ward identities, the structure of short multiplets and their 3 -point functions. In section 3 we outline the main proof of the nonrenormalization theorem in general context. In section 4 we provide a detailed proof of the theorem for $2 \mathrm{~d} \mathcal{N}=(4,4)$ SCFTs. In section 5 we present a detailed proof of the theorem for $4 \mathrm{~d} \mathcal{N}=4$ theories. In the remaining sections and appendices we provide various additional details.

\section{Background material}

In this section we review the basic ingredients that go into the proof of the nonrenormalization theorem. The reader who is familiar with basic properties of superconformal field theories can skip directly to the next section.

\subsection{Conformal perturbation theory}

Our goal is to understand the coupling constant dependence of certain correlation functions. Changing a coupling constant $g$ in a CFT corresponds to deforming the CFT by an exactly marginal operator $\mathcal{O}$. Correlators in the deformed theory can be computed from integrated correlators in the undeformed CFT. We have schematically

$$
\frac{\partial}{\partial g}\left\langle\mathcal{O}_{1}\left(x_{1}\right) \ldots \mathcal{O}_{n}\left(x_{n}\right)\right\rangle \sim \int d^{d} x\left\langle\mathcal{O}(x) \mathcal{O}_{1}\left(x_{1}\right) \ldots \mathcal{O}_{n}\left(x_{n}\right)\right\rangle
$$

This is only schematic because the integral has to be regularized due to UV divergences when $x$ approaches the other insertions. Because of these divergences and the need for regularization, marginal deformations at second order do not commute, we refer the reader to [18-20] for more details. ${ }^{3}$ Physically this can be understood as a certain kind of operator mixing: under marginal deformations there is an ambiguity of coupling-constant dependent redefinitions of operators with the same quantum numbers.

The picture that we should keep in mind is that in general the moduli space $\mathcal{M}$ (i.e. the space of marginal couplings of the CFT — sometimes called the "conformal manifold")

\footnotetext{
${ }^{3}$ An alternative approach is to attribute this phenomenon to the presence of "contact terms", as explained in $[21,22]$. Instead, the point of view we are adopting is that CFT correlators are only defined at distinct points and hence "contact terms" play absolutely no role. From this point of view operator mixing comes from the definition of the regularized integrated correlators, as was nicely discussed in [19]. The two approaches are equivalent, but we find it conceptually more clear to follow [19] and to avoid talking about contact terms. Hence, in the entirety of this paper we will never bring two local operators on the same spacetime point.
} 
is a higher dimensional manifold and the local operators of the CFT are sections of vector bundles over $\mathcal{M}$. So more precisely instead of (2.1), what we have is that

$$
\nabla_{g}\left\langle\mathcal{O}_{1}\left(x_{1}\right) \ldots \mathcal{O}_{n}\left(x_{n}\right)\right\rangle \equiv \int d^{d} x\left\langle\mathcal{O}(x) \mathcal{O}_{1}\left(x_{1}\right) \ldots \mathcal{O}_{n}\left(x_{n}\right)\right\rangle
$$

In general $\left[\nabla_{g_{1}}, \nabla_{g_{2}}\right] \neq 0$, which expresses that there is non-trivial operator mixing over the moduli space. The bundles on which operators take values have have non-trivial connection which enters this covariant derivative.

In this paper we will prove that certain correlation functions of chiral primaries do not depend on the couplings of the CFT. More precisely, what we need to show is that the covariant derivative of such correlators with respect to the couplings is zero. This is the "covariant" way to phrase the non-renormalization of correlation functions, which is unambiguous with respect to coupling constant dependent operator redefinitions.

Actually we will prove a stronger statement. We will not only show that - in certain supersymmetric CFTs, and for specific choices of the operators $\mathcal{O}_{1} \ldots \mathcal{O}_{n}-$ the r.h.s. of (2.2) vanishes, but we will show that the integrand on the r.h.s. of (2.2) is zero. This is a sufficient condition for the l.h.s. to vanish. The integral is supposed to be carefully regularized, and the operators are never brought on top of each other, so there is no subtlety with possible "contact terms" (see also footnote 3 ).

Let us then emphasize once more that if

$$
\left\langle\mathcal{O}(x) \mathcal{O}_{1}\left(x_{1}\right) \ldots \mathcal{O}_{n}\left(x_{n}\right)\right\rangle=0
$$

for distinct points, then it is guaranteed that the correlator $\left\langle\mathcal{O}_{1}\left(x_{1}\right) \ldots \mathcal{O}_{n}\left(x_{n}\right)\right\rangle$ does not change under marginal deformations by $\mathcal{O}$.

\subsection{Superconformal Ward identities}

For a general strongly coupled CFT there is no reason to expect the vanishing of a correlator of the form (2.3). The simplest reason for a correlator to exactly vanish is because of a symmetry of the theory. For example, if the CFT has an (unbroken) global U(1) symmetry, then a correlator is automatically zero if the charges of the inserted operators do not satisfy $\sum q_{i}=0$. In a sense, our proof will be based on similar conservation conditions, coming from the supersymmetric (and superconformal) charges of the theory.

Symmetries in CFTs are expressed in terms of Ward identities. In the case of a global internal symmetry with a conserved current $J_{\mu}$ we define the charge as $R=\int d^{d-1} x J_{0}(x)$ and then we can show that for any set of local operators we have

$$
\sum_{i=1}^{n}\left\langle\mathcal{O}_{1}\left(x_{1}\right) \ldots\left[R, \mathcal{O}_{i}\right]\left(x_{i}\right) \ldots \mathcal{O}_{n}\left(x_{n}\right)\right\rangle=0
$$

For global internal symmetries, this is the only type of Ward identity that we have.

The situation is richer for conserved "currents" with additional spacetime indices. For example, let us consider the stress energy tensor which satisfies $\partial^{\mu} T_{\mu \nu}=0$. Consider an arbitrary vector field $V_{\mu}(x)$ and construct the operator $j_{\mu}^{V}(x)=V^{\nu}(x) T_{\mu \nu}(x)$. Using that 
$T_{\mu \nu}$ is conserved and symmetric we have that $\partial^{\mu} j_{\mu}^{V}(x)=\frac{1}{2}\left(\partial^{\mu} V^{\nu}+\partial^{\nu} V^{\mu}\right) T_{\mu \nu}$. Combining this with the tracelessness of $T_{\mu \nu}$ we conclude that any vector field which satisfies $\partial^{\mu} V^{\nu}+$ $\partial^{\nu} V^{\mu}=\omega(x) g^{\mu \nu}$ leads to a conserved current $j_{\mu}^{V}$. Of course this is the condition for a conformal Killing vector field. Provided that $V_{\mu}(x)$ does not grow too fast at infinity, this can be used to define corresponding charges $R^{V}=\int d^{d-1} x j_{0}^{V}(x)$ and corresponding Ward identities, characterized by the choice of $V$. These conformal Ward identities are slightly more complicated than the ones for global internal symmetries, but are of course very well understood.

In this paper we will mostly use the superconformal Ward identities, i.e. the identities that follow from the existence of a supercurrent operator in the CFT. This is an operator of dimension $d-\frac{1}{2}$ and two Lorentz indices, a vector index $\mu$ and a spinor index $a$. Let us denote this operator as $G_{\mu a}$. We can construct (fermionic) conserved currents out of the supercurrent by contracting it with a spinor valued field $\psi^{a}(x)$ as

$$
j_{\mu}^{\psi}(x)=\psi^{a}(x) G_{\mu a}(x)
$$

The condition for $j_{\mu}^{\psi}$ to be conserved is that $\psi^{a}(x)$ must be a conformal Killing spinor. We also need to impose that it does not grow too fast as $|x| \rightarrow \infty$ in order for the corresponding charge $\int d^{d-1} x j_{0}^{\psi}(x)$ to be well-defined. Then we find the following possibilities. The first possibility is to take $\psi^{a}(x)$ to be a constant spinor independent of $x$. Then the charges $\int j_{0}^{\psi}$ are the usual supercharges that we denote by $\mathbf{Q}$. The second possibility is to take $\psi^{a}(x)$ to be linear in $x$ and then the corresponding charges turn out to be the "superconformal partners" of $\mathbf{Q}$ that we denote by $\mathbf{S} .{ }^{4}$ For a general conformal Killing spinor $\psi^{a}(x)$ which grows at most linearly at infinity the Ward identities have the schematic form

$$
\sum_{i} \psi\left(x_{i}\right)\left\langle\mathcal{O}_{1}\left(x_{1}\right) \ldots\left[\mathbf{Q}, \mathcal{O}_{i}\right\}\left(x_{i}\right) \ldots \mathcal{O}_{n}\left(x_{n}\right)\right\rangle+\psi^{\prime}\left(x_{i}\right)\left\langle\mathcal{O}_{1}\left(x_{1}\right) \ldots\left[\mathbf{S}, \mathcal{O}_{i}\right\}\left(x_{i}\right) \ldots \mathcal{O}_{n}\left(x_{n}\right)\right\rangle=0
$$

Here we have not shown explicitly the spinor indices of $\psi$ and how they are contracted with the supercharges in order not to clutter the notation. Also by $[\ldots, \ldots\}$ we mean commutator or anticommutator depending on whether the operator $\mathcal{O}$ is bosonic or fermionic.

It is important to notice that we can always choose $\psi^{a}(x)$ to vanish at some particular point $x_{i}$ and then the corresponding term proportional to $[\mathbf{Q}, \mathcal{O}\}\left(x_{i}\right)$ does not contribute to the Ward identity. This observation is quite crucial and it is a basic fact on which our proof is based. Notice also that if the operators $\mathcal{O}$ are superconformal primaries, we have $[\mathbf{S}, \mathcal{O}\}\left(x_{i}\right)=0$ and the Ward identity becomes particularly simple.

Notice a possible confusing point in the expression above. The commutators appearing in the Ward identity we are using are of the form $[\mathbf{Q}, \mathcal{O}](x)$, and not $[\mathbf{Q}, \mathcal{O}(x)]$. The first expression corresponds to first computing the commutator between $\mathbf{Q}$ and $\mathcal{O}(0)$ and then

\footnotetext{
${ }^{4}$ Our notation in this section is rather loose. By $\mathbf{S}$ we simply mean the "superconformal partner" of $\mathbf{Q}$ in the sense that they both come from the same supercurrent. In two dimensional notation we would have that $\mathbf{Q} \sim G_{-\frac{1}{2}}$ while $\mathbf{S} \sim G_{+\frac{1}{2}}$. In 4 d SCFTs if by $\mathbf{Q}$ we denote one of the left-chiral supercharges $Q_{a}$ then the corresponding $\mathbf{S}$ which comes from the same supercurrent is right-chiral $\mathbf{S} \sim \bar{S}_{\dot{a}}$. We hope the notation is not too confusing - more details on the superconformal Ward identities for $4 \mathrm{~d}$ SCFTs can be found in $[17,20]$.
} 
using the translation operators to translate the result at $x$, while the second expression is the commutator between $\mathbf{Q}$ and the translated operator $\mathcal{O}(x)$.

These two expression can be different. For example, if $\mathcal{O}$ is a superconformal primary, the commutator $[\mathbf{S}, \mathcal{O}(x)]$ vanishes only at the origin while $[\mathbf{S}, \mathcal{O}](x)$ clearly vanishes everywhere.

\subsection{Chiral primary 3-point functions}

Now we come to the correlators, whose non-renormalization we aim to prove. These are 3point functions of chiral primary operators i.e. operators belonging to "short" multiplets of the superconformal algebra. In theories with extended supersymmetry such operators must fall into representations of the non-abelian R-symmetry. For example, in the $\mathcal{N}=4 \mathrm{SYM}$ the R-symmetry is $\mathrm{SU}(4)$ while in $2 \mathrm{~d}$ CFTs with $\mathcal{N}=(4,4)$ it is $\mathrm{SU}(2)_{L} \otimes \mathrm{SU}(2)_{R}$. Hence the chiral primary operators are labeled by the representation $\mathcal{R}$ of the R-symmetry and also by a set of additional indices $\vec{m}$ that denote the specific element of the representation. As we will explain later, the general structure of the 3-point function is

$$
\left\langle\phi_{I}^{\left(\mathcal{R}_{1}, \vec{m}_{1}\right)}\left(x_{1}\right) \phi_{J}^{\left(\mathcal{R}_{2}, \vec{m}_{2}\right)}\left(x_{2}\right) \phi_{K}^{\left(\mathcal{R}_{3}, \vec{m}_{3}\right)}\left(x_{3}\right)\right\rangle=C_{I J K} \times(\text { group theoretic factors })
$$

where the indices $I, J, K$ label various irreducible representations of the R-symmetry group. The only dynamical information is in the coefficients $C_{I J K}$, which are precisely the coefficients whose independence of the coupling we need to prove. The "group theoretic factors" above contain both R-symmetry related factors, as well as the $x$-dependence of the correlator which is completely fixed by conformal invariance.

Given the general form (2.5) of these 3-point functions it becomes clear that we can isolate the desired coefficient $C_{I J K}$ by evaluating the correlator for specific alignments of the $\vec{m}$ 's, as long as the corresponding group theoretic factor is non-zero. In particular as we will explain in more detail later - it is possible to choose the operator at $x_{2}$ to be a "highest weight" state in the representation $\mathcal{R}_{2}$ and the one at $x_{3}$ to be a "lowest weight" state in $\mathcal{R}_{3}$, while the one at $x_{1}$ will be "mixed" i.e. will have weight $\vec{m}$ which is neither highest nor lowest. So we have that

$$
C_{I J K} \sim\left\langle\phi_{I}^{\left(\mathcal{R}_{1}, \vec{m}\right)}\left(x_{1}\right) \phi_{J}^{\left(\mathcal{R}_{2},+\right)}\left(x_{2}\right) \phi_{K}^{\left(\mathcal{R}_{3},-\right)}\left(x_{3}\right)\right\rangle
$$

where,+- denote the highest and lowest weight state respectively.

The constant of proportionality depends on group theoretic factors and is not relevant for us - as long as it is non-zero. Also notice that from the point of view of chiral primaries in $\mathcal{N}=1$ theories, the operator at $x_{2}$ would be "chiral primary", the one at $x_{3}$ would be "anti-chiral primary" while the one at $x_{1}$ would be neither chiral nor anti-chiral.

\subsection{Null vectors in short multiplets}

Before we proceed we need to make one more observation. The highest weight state of a short representation is annihilated by some of the supercharges. The lowest weight state is annihilated by the conjugate supercharges. However, "intermediate" weight states in short representations are generally not annihilated by any of the supercharges. 
While they are not annihilated by supercharges, these intermediate states satisfy "nullness conditions", by which we mean that certain linear combinations of superconformal descendants of intermediate weight states in the multiplet are zero. These can be derived by starting with the nullness conditions of the highest weight state $\left[\mathbf{Q}, \phi^{(\mathcal{R},+)}\right\}=0$ and acting on it with lowering operators of the R-symmetry algebra. Using the Jacobi identity these operators act both on the $\mathbf{Q}$ and on the chiral primary. Acting with such lowering operators repeatedly we get conditions which have the following general form

$$
\left[\mathbf{Q}, \phi^{(\mathcal{R}, \vec{m})}\right\}=\sum_{i} c_{i}\left[\mathbf{Q}_{i}^{\prime}, \phi^{\left(\mathcal{R}, \vec{m}_{i}^{\prime}\right)}\right\}
$$

where $\mathbf{Q}_{i}^{\prime}$ are supercharges with $\mathbf{R}$-symmetry weights different from those of $\mathbf{Q}$ and of course some of the $c_{i}$ 's may be zero. The operators $\phi^{\left(\mathcal{R}, \vec{m}_{i}^{\prime}\right)}$ are in the same multiplet as $\phi^{(\mathcal{R}, \vec{m})}$ but have different R-symmetry weight.

This condition will perhaps become more clear once we study it in specific theories.

\subsection{Supersymmetric marginal deformations}

The final element that we need is that the marginal deformations that we are interested in are of special kind, they are deformations that preserve not only conformal invariance but also supersymmetry. Imposing that superconformal invariance is preserved implies that the marginal operator must be a descendant of an (anti)-chiral primary. Let us illustrate this with a few examples.

In $2 \mathrm{~d} \mathcal{N}=(2,2)$ theories, the supersymmetric marginal deformations are of the form $^{5}\left\{\mathbf{G}_{-\frac{1}{2}}^{-},\left[\overline{\mathbf{G}}_{-\frac{1}{2}}^{-}, \phi\right]\right\}$ and $\left\{\mathbf{G}_{-\frac{1}{2}}^{+},\left[\overline{\mathbf{G}}_{-\frac{1}{2}}^{+}, \bar{\phi}\right]\right\}$ where $\phi, \bar{\phi}$ are chiral primaries in the $(c, c)$ and $(a, a)$ rings respectively, with conformal dimension $\left(\frac{1}{2}, \frac{1}{2}\right)$, and also of the form $\left\{\mathbf{G}_{-\frac{1}{2}}^{-},\left[\overline{\mathbf{G}}_{-\frac{1}{2}}^{+}, \psi\right]\right\}$ and $\left\{\mathbf{G}_{-\frac{1}{2}}^{+},\left[\overline{\mathbf{G}}_{-\frac{1}{2}}^{-}, \bar{\psi}\right]\right\}$ where $\psi, \bar{\psi}$ are chiral primaries in the $(a, c)$ and $(c, a)$ rings respectively, again with conformal dimension $\left(\frac{1}{2}, \frac{1}{2}\right)$.

Another example is the $\mathcal{N}=4 \mathrm{SYM}$ in $4 \mathrm{~d}$. There is only one (complex) marginal coupling $\mathcal{O}_{\tau}$ preserving the full $\mathcal{N}=4$ supersymmetry, corresponding to changes of the complexified gauge coupling $\tau=\frac{\theta}{2 \pi}+i \frac{4 \pi}{g^{2}}$. The moduli space of this CFT is the upper half $\tau$ plane, modded out by the appropriate S-duality group. The operator $\mathcal{O}_{\tau}$ is the (holomorphic part of the) Lagrangian density. The important thing for us is that it can be written as

$$
\mathcal{O}_{\tau}=\left\{\mathbf{Q},\left[\mathbf{Q},\left\{\mathbf{Q},\left[\mathbf{Q}, \operatorname{Tr}\left(Z^{2}\right)\right]\right\}\right]\right\}
$$

where $Z$ is one of the complex adjoint scalars. Here we did not write explicitly the indices of the supercharges - details can be found in appendix B.2. Notice that these supercharges are all of the same chirality so they (anti)-commute and their order is not important.

Instead of giving more examples, let us emphasize the main point: supersymmetric marginal operators can be written as

$$
\mathcal{O}=\{\mathbf{Q}, \Lambda\}
$$

\footnotetext{
${ }^{5}$ Here the G's are the supercharges which will be defined in more detail section 4 .
} 
where $\Lambda$ is some operator and $\mathbf{Q}$ is a supercharge which annihilates either highest, or lowest weight states. The operator $\Lambda$ is a descendant of chiral primaries of specific conformal dimension (the details depend on the theory).

Finally, let us remind that the marginal operator has to be a singlet of the R-symmetry of the theory. If not, it would break part of the supersymmetry.

\section{Outline of the proof}

Now we have collected all the ingredients and we can put them together to give an outline of the proof. The (theory-specific) details will be presented in the next sections.

Step 1: We isolate the dynamical part of the 3-point function by aligning the chiral primaries so that one of them is highest weight, the other lowest and the third intermediate. So we have

$$
C_{I J K} \sim\left\langle\phi_{I}^{\left(\mathcal{R}_{1}, \vec{m}\right)}\left(x_{1}\right) \phi_{J}^{\left(\mathcal{R}_{2},+\right)}\left(x_{2}\right) \phi_{K}^{\left(\mathcal{R}_{3},-\right)}\left(x_{3}\right)\right\rangle
$$

Step 2: We write the marginal operator corresponding to the change of a marginal coupling $g$ as $\mathcal{O}=\{\mathbf{Q}, \Lambda\}$. Hence we would like to prove the vanishing of

$$
\nabla_{g} C_{I J K} \sim \int d^{d} x\left\langle\{\mathbf{Q}, \Lambda\}(x) \phi_{I}^{\left(\mathcal{R}_{1}, \vec{m}\right)}\left(x_{1}\right) \phi_{J}^{\left(\mathcal{R}_{2},+\right)}\left(x_{2}\right) \phi_{K}^{\left(\mathcal{R}_{3},-\right)}\left(x_{3}\right)\right\rangle
$$

Let us denote the integrand by $\mathcal{I}$, on which we now focus.

Step 3: Without loss of generality we can assume that $\mathbf{Q}$ annihilates the highest weight operator at $x_{2}$. Then we use the superconformal Ward identity (2.4) with a spinor $\psi^{a}(x)$ vanishing at $x_{3}$ to move $\mathbf{Q}$ away from the point $x$ and we get that ${ }^{6}$

$$
\mathcal{I} \sim\left\langle\Lambda(x)\left[\mathbf{Q}, \phi_{I}^{\left(\mathcal{R}_{1}, \vec{m}\right)}\right\}\left(x_{1}\right) \phi_{J}^{\left(\mathcal{R}_{2},+\right)}\left(x_{2}\right) \phi_{K}^{\left(\mathcal{R}_{3},-\right)}\left(x_{3}\right)\right\rangle
$$

The important point here is that there is no other contribution to the Ward identity. ${ }^{7}$

Step 4: We use the "nullness condition" (2.6) for the operator at $x_{1}$ to rewrite this as

$$
\mathcal{I} \sim \sum_{,}\left\langle\Lambda(x)\left[\mathbf{Q}^{\prime}, \phi_{I}^{\left(\mathcal{R}_{1}, \vec{m}^{\prime}\right)}\right\}\left(x_{1}\right) \phi_{J}^{\left(\mathcal{R}_{2},+\right)}\left(x_{2}\right) \phi_{K}^{\left(\mathcal{R}_{3},-\right)}\left(x_{3}\right)\right\rangle
$$

where $\vec{m}^{\prime}$ is some other element of the same representation and $\mathbf{Q}^{\prime}$ supercharges with R-symmetry weight different from those of $\mathbf{Q}$.

Step 5: The set of supercharges $\mathcal{A}$ can be partitioned into two disjoint sets $\mathcal{A}=\mathcal{A}_{+} \cup \mathcal{A}_{-}$, where the charges in $\mathcal{A}_{+}$annihilate the highest weight states and the charges in $\mathcal{A}_{-}$ annihilate the lowest weight states. If $\mathbf{Q}^{\prime} \in \mathcal{A}_{+}$then we use the Ward identity with a

\footnotetext{
${ }^{6}$ Again, by $[\ldots, \ldots\}$ we mean the commutator (or anticommutator) if the operator is bosonic (or fermionic).

${ }^{7}$ Notice that $\phi_{I}, \phi_{J}, \phi_{K}$ are all superconformal primaries, so they are annihilated by the $\mathbf{S}$ 's.
} 
spinor vanishing at $x_{3}$ to move $\mathbf{Q}^{\prime}$ away from $x_{1}$. If $\mathbf{Q}^{\prime} \in \mathcal{A}_{-}$then we choose a spinor which vanishes at $x_{2}$. In both cases we have

$$
\mathcal{I} \sim \sum_{,}\left\langle\left\{\mathbf{Q}^{\prime}, \Lambda\right\}(x) \phi_{I}^{\left(\mathcal{R}_{1}, \vec{m}^{\prime}\right)}\left(x_{1}\right) \phi_{J}^{\left(\mathcal{R}_{2},+\right)}\left(x_{2}\right) \phi_{K}^{\left(\mathcal{R}_{3},-\right)}\left(x_{3}\right)\right\rangle
$$

Step 6: Remarkably the quantum numbers conspire in such a way that in the theories that we study $\left\{\mathbf{Q}^{\prime}, \Lambda\right\}=0$. Hence

$$
\mathcal{I}=0 \quad \Rightarrow \quad \nabla_{g} C_{I J K}=0
$$

This completes the proof.

Here we have skipped many details which will be presented in the next sections, since they are theory-dependent.

\section{Two-dimensional CFTs with $\mathcal{N}=(4,4)$ supersymmetry}

In this section we present the non-renormalization theorem for 3-point functions of chiral primaries in two-dimensional $\mathcal{N}=(4,4)$ superconformal field theories, generalizing and completing the results of [18].

In the first subsection we describe the short multiplets in these theories and review the general form of the 3-point function of chiral primaries. In the second subsection we prove the non-renormalization theorem.

\subsection{Short representations and their 3-point functions}

The R-symmetry of the $\mathcal{N}=(4,4)$ superconformal algebra is $\mathrm{SU}(2)_{L} \otimes \mathrm{SU}(2)_{R}$. The left moving supercharges are denoted by $\mathbf{G}_{-\frac{1}{2}}^{a r}$. Here the index $a= \pm$ denotes the $J^{3}$ eigenvalue with respect to the left-moving $\mathrm{SU}_{2}(2)_{L}$ R-symmetry, while the index $r= \pm$ denotes the eigenvalue of the supercharge under a left $\mathrm{SU}(2)$ outer automorphism of the $\mathcal{N}=4$ algebra. The right-moving supercharges have similar structure. We refer the reader to [18] for more details.

Representations of the algebra are labeled by the conformal dimension $\{h, \bar{h}\}$ and the R-symmetry representation $\{j, \bar{j}\}$ of the superconformal primaries ${ }^{8}$ of the multiplet. Notice that a given multiplet contains several superconformal primaries which differ by their $\mathrm{SU}(2)_{L} \otimes \mathrm{SU}(2)_{R}$ quantum numbers. Unitarity requires

$$
h \geq j, \quad \bar{h} \geq \bar{j}
$$

Multiplets which saturate the bound are "short" and are usually called "chiral primary" multiplets.

To simplify notation, in the following we will sometimes write only the quantum numbers of the left-moving sector. For a multiplet characterized by conformal dimension $h$ and R-symmetry quantum number $j$, we have the following set of superconformal primaries

${ }^{8}$ I.e. operators annihilated by all $\mathbf{G}_{n}^{a b}, n>0$.

$$
\phi^{(j, m)}, \quad m=-j, \ldots,+j
$$


which differ by their $J^{3}$ eigenvalue $m$. All these operators are superconformal primaries, they have conformal dimension $h$ and can be recovered from the "highest weight" state of the multiplet by acting with $\mathrm{SU}(2)_{L}$ lowering operators

$$
\phi^{(j, m)} \sim \overbrace{\left[J^{-}, \ldots\left[J^{-}\right.\right.}^{j-m}, \phi^{(j, j)}] \ldots]
$$

The "highest weight" operator of a short multiplet $\phi^{(j, j)}$ is annihilated by some of the supercharges

$$
\left[\mathbf{G}_{-\frac{1}{2}}^{+r}, \phi^{(j, j)}\right\}=0, \quad r=+,-
$$

and similarly for the "lowest weight" one $\phi^{(j,-j)}$

$$
\left[\mathbf{G}_{-\frac{1}{2}}^{-r}, \phi^{(j,-j)}\right\}=0, \quad r=+,-
$$

The other members of the short multiplet $\phi^{(j, m)}$ with $m \neq \pm j$ are not annihilated by any of the left moving supercharges. They do however satisfy nullness conditions, which can be derived by starting with $\left[\mathbf{G}_{-\frac{1}{2}}^{+r}, \phi_{I}^{(j, j)}\right\}=0$ and acting with lowering operators $J^{-}$. This leads to the following relation ${ }^{9}$

$$
\left[\mathbf{G}_{-\frac{1}{2}}^{+r}, \phi_{I}^{(j, n)}\right\} \sim\left[\mathbf{G}_{-\frac{1}{2}}^{-r}, \phi_{I}^{(j, n+1)}\right\}
$$

where the constant of proportionality is nonzero as long as $n<j$.

Notice that here there is some potentially confusing terminology: from the $\mathcal{N}=(4,4)$ point of view, all the operators $\phi^{(j, m)}$ are sometimes called "chiral primaries", since they all belong to the same short multiplet. If however we consider an $\mathcal{N}=(2,2)$ subalgebra then the operator $\phi^{(j, j)}$ would be called "chiral", the operator $\phi^{(j,-j)}$ "antichiral" and the other operators $\phi^{(j, m)}$ with $m \neq \pm j$ would be neither chiral nor antichiral.

Let us write the general form of the 3-point function of chiral primary operators. We have

$$
\begin{aligned}
\left\langle\phi_{I}\left(x_{1}\right)\right. & \left.\phi_{J}\left(x_{2}\right) \phi_{K}\left(x_{3}\right)\right\rangle=C_{I J K}\left(\begin{array}{ccc}
j_{1} & j_{2} & j_{3} \\
m_{1} & m_{2} & m_{3}
\end{array}\right)\left(\begin{array}{ccc}
\bar{j}_{1} & \bar{j}_{2} & \bar{j}_{3} \\
\bar{m}_{1} & \bar{m}_{2} & \bar{m}_{3}
\end{array}\right) \\
& \times \frac{1}{x_{12}^{\left(j_{1}+j_{2}-j_{3}\right)} x_{23}^{\left(j_{2}+j_{3}-j_{1}\right)} x_{13}^{\left(j_{1}+j_{3}-j_{2}\right)}} \frac{1}{\bar{x}_{12}^{\left(\bar{j}_{1}+\bar{j}_{2}-\bar{j}_{3}\right)} \bar{x}_{23}^{\left(\bar{j}_{2}+\bar{j}_{3}-\bar{j}_{1}\right)} \bar{x}_{13}^{\left(\bar{j}_{1}+\bar{j}_{3}-\bar{j}_{2}\right)}}
\end{aligned}
$$

Here we did not write explicitly the $\mathrm{SU}(2)_{L} \otimes \mathrm{SU}(2)_{R}$ quantum numbers on the 1.h.s. of the equation.

The $x$-dependence in (4.2) is fixed by conformal invariance in terms of the conformal dimension of the operators. The dependence on the quantum numbers $(j, m ; \bar{j}, \bar{m})$ is fixed by the $\mathrm{SU}(2)_{L} \otimes \mathrm{SU}(2)_{R}$ R-symmetry and is expressed by the 3 -j symbols presented above. All the dynamical information is encoded in the coefficient $C_{I J K}$, which as we can see only depends on the choice of chiral primary representations $I, J, K$ and not on the specific

\footnotetext{
${ }^{9}$ This equation is proven in appendix $\mathrm{C}$.
} 
representatives from each of them (i.e. does not depend on the R-symmetry quantum numbers $m, \bar{m})$.

Our goal is to show that the constants $C_{I J K}$ do not depend on the coupling constants of the CFT.

Going back to the distinction between a "chiral primary" in $\mathcal{N}=(2,2)$ theories and a "chiral primary" in $\mathcal{N}=(4,4)$ theories let us notice the following: in $\mathcal{N}=(2,2)$ theories $\mathrm{R}$-charge conservation requires that the three operators satisfy the condition $j_{3}=j_{1}+j_{2}$ (or permutations) - and similarly for the right-moving sector. These would be "extremal" 3 -point functions of chiral primaries from the $\mathcal{N}=(4,4)$ point of view. However in $\mathcal{N}=$ $(4,4)$ theories there are also 3 -point functions of chiral primaries which are not in the extremal case.

In [18] a non-renormalization theorem for 3-point functions was proven for the special case where the three chiral primary multiplets satisfy $j_{3}=j_{1}+j_{2}$ (or permutations) i.e. for the "extremal case". In that case the 3-point function can be viewed as a 3-point function of chiral primaries of an $\mathcal{N}=(2,2)$ subalgebra. In the more general case where $j_{3} \neq j_{1}+j_{2}$ this is not possible. There is no way to align all three operators so that they are all in the chiral ring of a given $\mathcal{N}=(2,2)$ subalgebra. Nevertheless, the "non-extremal" 3 -point functions also seem to be protected and thus should obey some non-renormalization theorem, which we will prove in the next subsection.

\subsection{The non-renormalization theorem in $2 \mathrm{~d}$}

Theories with $\mathcal{N}=(4,4)$ supersymmetry in two dimensions have a moduli space of marginal deformations which is locally of the form $\frac{\mathrm{SO}(n, 4)}{\mathrm{SO}(n) \times \mathrm{SO}(4)}[21]$. Here $n$ is the number of chiral primary multiplets which transform in the $(1 / 2,1 / 2)$ representation of the $\mathrm{SU}(2)_{L} \otimes \mathrm{SU}(2)_{R}$ R-symmetry group.

Let us consider the 3-point function of operators which belong to chiral primary multiplets

$$
\left\langle\phi_{I}\left(x_{1}\right) \phi_{J}\left(x_{2}\right) \phi_{K}\left(x_{3}\right)\right\rangle
$$

where for simplicity we do not write any R-symmetry indices, this is supposed to be a condensed notation for (4.2). Let us also consider a marginal operator $\mathcal{O}$ corresponding to the change of a marginal coupling constant $g$. By definition we have

$$
\nabla_{g}\left\langle\phi_{I}\left(x_{1}\right) \phi_{J}\left(x_{2}\right) \phi_{K}\left(x_{3}\right)\right\rangle \equiv \int d^{2} x\left\langle\mathcal{O}(x) \phi_{I}\left(x_{1}\right) \phi_{J}\left(x_{2}\right) \phi_{K}\left(x_{3}\right)\right\rangle
$$

In order to prove that the 3-point functions are independent of the coupling we have to show that the expression above vanishes. We will actually prove a stronger statement, namely that

$$
\mathcal{I} \equiv\left\langle\mathcal{O}(x) \phi_{I}\left(x_{1}\right) \phi_{J}\left(x_{2}\right) \phi_{K}\left(x_{3}\right)\right\rangle=0
$$

even without integrating over $x$. We will follow the steps outlined in section 3 .

In order to prove this we will use two properties of the $\mathcal{N}=(4,4)$ algebra.

First, we exploit the $\mathrm{SU}(2)_{L} \otimes \mathrm{SU}(2)_{R}$ structure of the correlator (4.2). If we simply want to compute the 3 -point function $C_{I J K}$ - or rather to prove that it is independent of 
the coupling — we are free to evaluate the correlator for any alignment of the operators for which the $3 \mathrm{j}$ symbols are non-vanishing. Hence we will choose the representatives of the other chiral primaries in the following way

$$
\mathcal{I} \sim\left\langle\mathcal{O}(x) \phi_{I}^{\left(j_{1}, n\right)}\left(x_{1}\right) \phi_{J}^{\left(j_{2}, j_{2}\right)}\left(x_{2}\right) \phi_{K}^{\left(j_{3},-j_{3}\right)}\left(x_{3}\right)\right\rangle
$$

where $n=j_{3}-j_{2}$. The constant of proportionality is some (non-vanishing) group-theoretic factor which is of no interest for our argument. Notice that from the point of view of an $\mathcal{N}=2$ subalgebra the operator at $x_{2}$ is "chiral primary", the operator at $x_{3}$ is "anti-chiral primary" while the operator at $x_{1}$ is neither chiral on antichiral.

Second, without loss of generality ${ }^{10}$ we can assume that the marginal operator can be written as $\mathcal{O}=\left\{\mathbf{G}_{-\frac{1}{2}}^{+r},\left[\overline{\mathbf{G}}_{-\frac{1}{2}}^{+s}, \bar{\phi}\right]\right\}$ where $\bar{\phi}$ is an element of a chiral primary multiplet of conformal weight $\left(\frac{1}{2}, \frac{1}{2}\right)$ and which is aligned to have $\left(J^{3}, \bar{J}^{3}\right)=\left(-\frac{1}{2},-\frac{1}{2}\right)$.

Then we have that

$$
\mathcal{I} \sim\left\langle\left(\left\{\mathbf{G}_{-\frac{1}{2}}^{+r},\left[\overline{\mathbf{G}}_{-\frac{1}{2}}^{+s}, \bar{\phi}\right]\right\}\right)(x) \phi_{I}^{\left(j_{1}, n\right)}\left(x_{1}\right) \phi_{J}^{\left(j_{2}, j_{2}\right)}\left(x_{2}\right) \phi_{K}^{\left(j_{3},-j_{3}\right)}\left(x_{3}\right)\right\rangle
$$

Using a superconformal Ward identity (2.4) for $\mathbf{G}_{-\frac{1}{2}}^{+r}$ with a conformal Killing spinor vanishing at the point $x_{3}$ we find that this can be written as

$$
\mathcal{I} \sim\left\langle\left[\overline{\mathbf{G}}_{-\frac{1}{2}}^{+s}, \bar{\phi}\right](x)\left[\mathbf{G}_{-\frac{1}{2}}^{+r}, \phi_{I}^{\left(j_{1}, n\right)}\right\}\left(x_{1}\right) \phi_{J}^{\left(j_{2}, j_{2}\right)}\left(x_{2}\right) \phi_{K}^{\left(j_{3},-j_{3}\right)}\left(x_{3}\right)\right\rangle
$$

where the constant of proportionality in this expression is different from zero. Here we used that $\mathbf{G}_{-\frac{1}{2}}^{+r}$ annihilates the operator at $x_{2}$.

Now we use the nullness condition (4.1) to rewrite it as

$$
\mathcal{I} \sim\left\langle\left[\overline{\mathbf{G}}_{-\frac{1}{2}}^{+s}, \bar{\phi}\right](x)\left[\mathbf{G}_{-\frac{1}{2}}^{-r}, \phi_{I}^{\left(j_{1}, n+1\right)}\right\}\left(x_{1}\right) \phi_{J}^{\left(j_{2}, j_{2}\right)}\left(x_{2}\right) \phi_{K}^{\left(j_{3},-j_{3}\right)}\left(x_{3}\right)\right\rangle
$$

Finally we use a superconformal Ward identity for $\mathbf{G}_{-\frac{1}{2}}^{-r}$ with a conformal Killing spinor which vanishes at the point $x_{2}$. All other operators do not contribute because they are annihilated by $\mathbf{G}_{-\frac{1}{2}}^{-r}$, hence we find

$$
\mathcal{I}=0
$$

This proves that 3 -point functions of chiral primaries are independent of the coupling constant.

Notice that it would not be possible to apply a similar argument to prove nonrenormalization of 4- and higher point functions of chiral primaries (unless they are extremal [18], see also [23, 24]) - which is of course consistent, since we know that such correlators do depend on the coupling constants.

\section{Four-dimensional $\mathcal{N}=4$ SCFTs}

The same type of argument can be used to prove the non-renormalization of 3-point functions of $1 / 2$ BPS chiral primaries in four-dimensional SCFTs with $\mathcal{N}=4$ supersymmetry.

\footnotetext{
${ }^{10}$ This is a general property of $\mathcal{N}=(4,4)$ SCFTs which was discussed in detail in [18].
} 


\subsection{Short representations}

Now the R-symmetry is SU(4). We choose a basis for its Cartan subalgebra. The short representations that we are interested in are those with Dynkin labels $[0, k, 0]$, Lorentz spin $(j, \bar{j})=(0,0)$ and conformal dimension $\Delta=k$. These are the "1/2 BPS" operators of the $\mathcal{N}=4$ algebra. In terms of Young tableaux for $\mathrm{SU}(4)$ these representations correspond to tableaux with $k$ columns of length 2 (we refer to appendix B.1 for more details). As before we denote the superconformal primaries of such a multiplet by

$$
\phi^{(k, \vec{m})}
$$

where now $\vec{m}$ labels the weight of the state inside the SU(4) multiplet (i.e. $\vec{m}$ are the eigenvalues of the state under the Cartan generators). Of special interest will be the highest and lowest weight states of any given representation, which we call $\phi^{(k, \pm)}$. For example, in some conventions highest weight operators are $\operatorname{Tr}\left(Z^{k}\right)$ and their multi-trace products.

Let us recall some group theory (more details are given in appendix A and B). We denote by $E_{i}$ the generators of $\mathrm{SU}(4)$ corresponding to positive simple roots, or raising operators. The highest weight state satisfies $\left[E_{i}, \phi^{(k,+)}\right]=0$. Other operators in the same SU(4) multiplet can be recovered starting from $\phi^{(k,+)}$ and acting with the lowering operators $E_{i}^{\dagger}$

$$
\phi^{(k, \vec{m})} \sim\left[E_{i_{n}}^{\dagger}, \ldots\left[E_{i_{1}}^{\dagger}, \phi^{(k,+)}\right] \ldots\right]
$$

where the product is some specific combination of the "negative simple roots", perhaps with repeated appearances.

Of course equivalently we can start from the lowest weight state and get the same state by acting with "raising" operators.

$$
\phi^{(k, \vec{m})} \sim\left[E_{i_{n}}, \ldots\left[E_{i_{1}}, \phi^{(k,-)}\right] \ldots\right]
$$

It is a group-theoretic fact that in a tensor product of the form $\left[0, k_{1}, 0\right] \otimes\left[0, k_{2}, 0\right]$ any representation of the form $\left[0, k_{3}, 0\right]$ appears either one time or none. ${ }^{11}$ Hence the general form of a 3-point function is

$$
\begin{aligned}
\left\langle\phi_{I}^{\left(k_{1}, \vec{m}_{1}\right)}\left(x_{1}\right) \phi_{J}^{\left(k_{2}, \vec{m}_{2}\right)}\left(x_{2}\right) \phi_{K}^{\left(k_{3}, \vec{m}_{3}\right)}\left(x_{3}\right)\right\rangle= & C_{I J K} \mathbf{G}\left(k_{1}, \vec{m}_{1} ; k_{2}, \vec{m}_{2} ; k_{3}, \vec{m}_{3}\right) \\
& \times \frac{1}{\left|x_{12}\right|^{k_{1}+k_{2}-k_{3}}\left|x_{23}\right|^{k_{2}+k_{3}-k_{1}}\left|x_{13}\right|^{k_{1}+k_{3}-k_{2}}}
\end{aligned}
$$

where $\mathbf{G}\left(k_{1}, \vec{m}_{1} ; k_{2}, \vec{m}_{2} ; k_{3}, \vec{m}_{3}\right)$ is the (unique) SU(4) Clebsh-Gordan coefficient for three representations of the type $[0, k, 0]$ i.e. a group-theoretic factor. The dynamical information is encoded in the coefficient $C_{I J K}$.

Notice that, as emphasized previously in the paper, it is only the highest and lowest weight states of the short multiplets that are annihilated by supercharges. "Intermediate weight" states are generally not annihilated by any of the supercharges (though they lead to

\footnotetext{
${ }^{11}$ If $k_{1}, k_{2}, k_{3}$ satisfy the triangle (in)-equality and $\frac{k_{1}+k_{2}+k_{3}}{2}$ is an integer, then the representation appears one time. Otherwise it does not appear.
} 
certain "nullness conditions" as explained earlier). For example, while the superconformal primary operators of the form

$$
C_{i_{1} \ldots i_{n}} \operatorname{Tr}\left(\phi^{i_{1}} \cdots \phi^{i_{k}}\right)
$$

with $C$ symmetric and traceless are members of $1 / 2$ BPS multiplets, for generic choice of such symmetric traceless $C$, they are not annihilated by any supercharges. Only if $C$ is chosen so that the corresponding operator is highest or lowest weight state with respect to $\mathrm{SU}(4)_{R}$ is the operator annihilated by $1 / 2$ of the supercharges. ${ }^{12}$

\subsection{The non-renormalization theorem in $4 \mathrm{~d}$}

First let us choose a basis of the left chiral supercharges so that they have definite weight under the Cartan subalgebra. ${ }^{13}$ We denote these left chiral supercharges as $\mathbf{Q}_{a}^{i}$ where the index $i=1, \ldots 4$ is the $\mathrm{SU}(4)$ and $a$ the Lorentz index.

The theory has an exactly marginal operator $\mathcal{O}_{\tau}$ corresponding to the change of the complexified coupling constant $\tau=\frac{\theta}{2 \pi}+i \frac{4 \pi}{g^{2}}$. As mentioned before and explained in detail in appendix B. 2 - this operator can be written as

$$
\mathcal{O}_{\tau}=(\mathbf{Q})^{4} \phi^{(2,+)}
$$

where only four of the left-chiral supercharges act on the highest weight state. The notation $(\mathbf{Q})^{4}$ means the nested (anti)-commutator, as in equation (2.7), we hope this is obvious. Notice that the left chiral supercharges anticommute among themselves so we do not need to worry about the order with which they act on an operator.

The set of left chiral supercharges $\mathcal{A}$ can be partitioned into two disjoint sets $\mathcal{A}=\mathcal{A}_{+} \cup$ $\mathcal{A}_{-}$, where the charges in $\mathcal{A}_{+}$annihilate the highest weight states of the $1 / 2$ BPS multiplets and the charges in $\mathcal{A}_{-}$annihilate the lowest weight states. The set of supercharges which appear in (5.2) is simply $\mathcal{A}_{-}$, and any other left chiral supercharge in $\mathcal{A}_{+}$annihilates the operator $\phi^{(2,+)}$. This will be important below.

Consider now the change of a 3-point function under a deformation by $\mathcal{O}_{\tau}$. We will show that

$$
\mathcal{I} \equiv\left\langle\mathcal{O}_{\tau}(x) \phi_{I}\left(x_{1}\right) \phi_{J}\left(x_{2}\right) \phi_{K}\left(x_{3}\right)\right\rangle=0
$$

As before we can choose the SU(4) alignment of the operators in such a way that

$$
\mathcal{I} \sim\left\langle\mathcal{O}_{\tau}(x) \phi_{I}^{\left(k_{1}, \vec{m}_{1}\right)}\left(x_{1}\right) \phi_{J}^{\left(k_{2},+\right)}\left(x_{2}\right) \phi_{K}^{\left(k_{3},-\right)}\left(x_{3}\right)\right\rangle
$$

where the operator at $x_{2}$ is a highest weight state, the one at $x_{3}$ is lowest weight and the one at $x_{1}$ is of some general weight in the representation $k_{1}$. Using the form of the marginal operator we have

$$
\mathcal{I} \sim\left\langle\left((\mathbf{Q})^{4} \phi^{(2,+)}\right)(x) \phi_{I}^{\left(k_{1}, \vec{m}_{1}\right)}\left(x_{1}\right) \phi_{J}^{\left(k_{2},+\right)}\left(x_{2}\right) \phi_{K}^{\left(k_{3},-\right)}\left(x_{3}\right)\right\rangle
$$

\footnotetext{
${ }^{12}$ In [17] it was incorrectly assumed that all superconformal primaries of the $1 / 2$ BPS multiplet are annihilated by half of the supercharges, hence the proposed proof of the non-renormalization theorem in $[17]$ is incomplete.

${ }^{13}$ And also a definite weight under the $J_{3}$ of the $\mathrm{SU}(2)_{L}$ part of the Lorentz group.
} 
Notice that the four supercharges acting on the operator at $x$ are all left chiral so they (anti)-commute and their order is not important. As we mentioned above we call this set of supercharges $\mathcal{A}_{-}$. Also notice that all of these four supercharges annihilate the operator at $x_{3}$.

We take one of them, let us call it $\mathbf{Q}^{\star}$ and move it away using the Ward identity. We choose the conformal Killing spinor to vanish at the point $x_{2}$. Hence the correlator becomes

$$
\mathcal{I} \sim\left\langle\left((\mathbf{Q})^{3} \phi^{(2,+)}\right)(x)\left(\left[\mathbf{Q}^{\star}, \phi_{I}^{\left(k_{1}, m_{1}\right)}\right]\right)\left(x_{1}\right) \phi_{J}^{\left(k_{2},+\right)}\left(x_{2}\right) \phi_{K}^{\left(k_{3},-\right)}\left(x_{3}\right)\right\rangle
$$

Now we will use the analogue of (4.1) coming from the fact that $\mathbf{Q}^{\star}$ annihilates the lowest weight state of the representation $k_{1}$, that is.

$$
\left[\mathbf{Q}^{\star}, \phi_{I}^{\left(k_{1}, \vec{m}_{1}\right)}\right]=\sum_{j \neq \star}\left[\mathbf{Q}^{j}, \mathcal{X}_{j}\right]
$$

where all supercharges in the sum on the r.h.s. are left chiral and different from $\mathbf{Q}^{\star}$ and $\mathcal{X}_{j}$ is either one of the elements of the multiplet $\phi_{I}^{\left(k_{1}, \vec{m}_{j}\right)}$ or perhaps zero. ${ }^{14}$ This important relation is proven in appendix $\mathrm{C}$.

Next, for each of these $\mathbf{Q}^{j}$ 's we apply the Ward identity (2.4) again. There are two possibilities:

1) $\mathbf{Q}^{j}$ is in $\mathcal{A}_{-}$: in this case we use (2.4) with a spinor vanishing at $x_{2}$. We do not get any contribution from $x_{3}$ because the operator is annihilated by the supercharges in $\mathcal{A}_{-}$. We do not get any contribution from $x$ because the supercharge is already there, so it squares to zero.

2) $\mathbf{Q}^{j}$ is in $\mathcal{A}_{+}$: then this supercharge annihilates operators of the form $\phi^{(k,+)}$. Then we use (2.4) superconformal Ward identity with a spinor vanishing at $x_{3}$ and we get zero.

So in all cases the contribution is zero. Hence

$$
\mathcal{I}=0 \quad \Rightarrow \quad \nabla_{\tau} C_{I J K}=0
$$

Exactly the same argument can be applied for the marginal operator $\overline{\mathcal{O}}_{\tau} \equiv(\overline{\mathbf{Q}})^{4} \phi^{(2,-)}$. So all in all the 3-point functions are not renormalized and this completes the proof. Notice that this argument fails - as expected - if we try to prove the non-renormalization of $n$ point functions of chiral primaries with $n>3$ (unless they are "extremal"). The last step of the proof relied on the fact that there was at most one operator which was not annihilated by the supercharge involved in the Ward identity. We chose the Killing spinor to vanish at the point where this operator was inserted. If there were more than one operators not annihilated by the supercharge, it would not be possible to simultaneously "hide" their contributions to the Ward identity by choosing the Killing spinor appropriately.

\footnotetext{
${ }^{14}$ In either case the operator $\mathcal{X}$ is annihilated by the $\bar{S}$ 's.
} 


\section{Extremal correlators}

Similar arguments can be used to show that a certain class of higher $n$-point functions are not renormalized. These are the so-called "extremal correlators" i.e. correlators where all chiral primaries are aligned to be "highest weight" except for one that is aligned to be "lowest weight" and which ensures R-charge neutrality

$$
\left\langle\phi_{1}^{\left(\mathcal{R}_{1},+\right)}\left(x_{1}\right) \phi_{2}^{\left(\mathcal{R}_{2},+\right)}\left(x_{2}\right) \ldots \phi_{n}^{\left(\mathcal{R}_{n},-\right)}\left(x_{n}\right)\right\rangle
$$

Charge conservation shows that the operators must satisfy $\Delta_{n}=\sum_{i=1}^{n-1} \Delta_{i}$.

That such correlators are not renormalized in $2 \mathrm{~d} \mathcal{N}=(4,4)$ theories was proven in [18]. The proof was based on the observation that in these theories a marginal operator can always be written as $\mathcal{O}=\left[\mathbf{G}_{-\frac{1}{2}}^{-r}, \Lambda\right]$. Then we can consider

$$
\left\langle\mathcal{O}(z) \phi_{1}^{\left(\mathcal{R}_{1},+\right)}\left(x_{1}\right) \phi_{2}^{\left(\mathcal{R}_{2},+\right)}\left(x_{2}\right) \ldots \phi_{n}^{\left(\mathcal{R}_{n},-\right)}\left(x_{n}\right)\right\rangle
$$

and use a Ward identity with a spinor vanishing at $x_{n}$ to move the supercharges away from $z$. The operators at $x_{1}, \ldots, x_{n-1}$ do not contribute since they are annihilated by $\bar{G}_{-\frac{1}{2}}^{-r}$ and the operator at $x_{n}$ does not contribute because of the choice of the spinor in the Ward identity. Hence this correlator vanishes and the desired result is proven.

Let us quickly repeat the similar statement in $\mathcal{N}=4 \mathrm{SYM}$. In that theory we have two marginal operators - corresponding to changes of the coupling constant $g$ and the $\theta$-angle - which can be combined into the holomorphic and anti-holomorphic operators $\mathcal{O}_{\tau}, \overline{\mathcal{O}}_{\tau}$. One of these operators can be written as

$$
\overline{\mathcal{O}}_{\tau}=(\overline{\mathbf{Q}})^{4} \operatorname{Tr}\left(\bar{Z}^{2}\right)
$$

where the supercharges $\overline{\mathbf{Q}}$ annihilate highest weight states of SU(4). Hence we can use the Ward identity with a spinor vanishing at $x_{n}$ to show that the analogue of (6.1) in $\mathcal{N}=4$ vanishes. To complete the proof of the non-renormalization we also need to show that the same correlator vanishes for the marginal operator $\mathcal{O}_{\tau}$. We can use the fact that in $\mathcal{N}=4$ theories this marginal operator can also be written as

$$
\mathcal{O}_{\tau}=(\mathbf{Q})^{4} \operatorname{Tr}\left(\bar{Z}^{2}\right)
$$

where the Q's are supercharges of left chirality. This may look confusing when compared to (6.2) and against our intuition from theories with less supersymmetry, but it is indeed a true statement (explained in appendix B.2). ${ }^{15}$ The four supercharges in (6.3) annihilate the highest weight states of SU(4) of the form $\phi^{(k,+)}$. Hence the Ward identities can be used as above to show that the correlator vanishes.

All in all we have proved that extremal $n$-point functions of $1 / 2$ BPS chiral primaries in four-dimensional $\mathcal{N}=4 \mathrm{SCFT}$ s are not renormalized.

\footnotetext{
${ }^{15}$ Notice that the four Q's in (6.3) are not the complex conjugates of the supercharges in (6.2).
} 


\section{Other extensions}

In this section we list some immediate generalizations of our results.

\subsection{Half-chiral states in $2 \mathrm{~d} \mathcal{N}=(4,4)$}

Interestingly, the argument in section 4 relied only on one sector - say the left moving one - of the CFT. This implies that the same argument goes through without changes when applied to 3-point functions of operators that are in short multiplets of the left-moving $\mathrm{SU}(2)_{L}$ and long multiplets on the right-moving one. Such operators are of the form (chiral, anything). Our argument shows that their 3-point functions are not renormalized as a function of the coupling constants. Notice that these states are related by spectral flow to states of the form (Ramond ground state, anything) which are precisely the microstates of the Strominger-Vafa black hole [25]. It would be interesting to explore the possible applications of this statement.

Notice however that our arguments show that the 3-point functions of such states do not renormalize as a function of the coupling assuming that they remain chiral primaries during the deformation (i.e. that short multiplets do not combine and lift from the BPS bound). We have not addressed the issue of whether BPS states lift or not under marginal deformations.

\subsection{3 -point functions in $2 \mathrm{~d} \mathcal{N}=(0,4)$ SCFTs}

Another interesting case is that of two-dimensional CFTs with $(0,4)$ supersymmetry. In string theory they arise on the worldvolume of bound states of M2/M5 branes wrapped on Calabi-Yau compactifications of M-theory and are relevant for the computation of the entropy of certain supersymmetric black holes [26, 27].

Theories with $\mathcal{N}=(0,4)$ supersymmetry are not very well understood, but it is clear that on their "supersymmetric side" they have operators in short representations, which are the analogue of the (anything, chiral) operators in $(4,4)$ CFTs. Our claim is that 3-point functions of such operators are not renormalized as a function of the coupling constants. This follows immediately from our proof, if we also remember that marginal operators in these theories can be written as $\mathcal{O}=\left[\bar{G}_{-\frac{1}{2}}^{ \pm r}, \phi\right]$ and its conjugate, where $\phi$ is "chiral primary" with respect to the right moving supersymmetric side. Also, notice that the statement holds only for operators which do not lift from the BPS bound as we vary the coupling.

\subsection{Less supersymmetric multiplets in $4 \mathrm{~d}$}

It would be interesting to generalize our results to $1 / 4$ and $1 / 8$ BPS operators in four dimensional $\mathcal{N}=4 \mathrm{SCFT}$. Unfortunately, the group theory structure of the correlators is much more intricate in this case. For example, the product of three $1 / 4$ BPS scalar operators, which sit in $[q, p, q]$ representations of the $\mathrm{SU}(4) \mathrm{R}$-symmetry group, contains many trivial representations. As an example, the product of three $[1,2,1]$ representations contains 5 distinct trivial representations. This means that the corresponding 3-point 
functions are not determined by a single numerical coefficient, unlike what happened in the $1 / 2$ BPS case.

As a consequence, the first step of choosing an alignment cannot be carried out in general. It is interesting to explore whether the rest of the proof extends at least for specific alignments. So let us consider a general 3-point function, aligned in a convenient way, and let us try to derive some necessary conditions for our proof to hold. It is clear that the highest-weight of such operators should be annihilated by at least one supercharge, so let us consider the product of three $1 / 8$ BPS operators, so that the change of their 3 -point function generated by $\mathcal{O}_{\tau}$ reads

$$
\mathcal{I} \sim\left\langle\left((\mathbf{Q})^{4} \phi^{(2,+)}\right)(x) \phi_{I}^{\left(\mathcal{R}_{1}, \vec{m}_{1}\right)}\left(x_{1}\right) \phi_{J}^{\left(\mathcal{R}_{2},+\right)}\left(x_{2}\right) \phi_{K}^{\left(\mathcal{R}_{3},-\right)}\left(x_{3}\right)\right\rangle
$$

The charges appearing in $(\mathbf{Q})^{4}$ are either $\mathbf{Q}^{3}$ or $\mathbf{Q}^{4}$. We can take one of the two ${ }^{16} \mathbf{Q}^{4}$ 's (which annihilates the operator at $x_{3}$, since it is a lowest-weight) and move it using a Ward identity with a conformal Killing spinor that vanishes at $x_{2}$ :

$$
\mathcal{I} \sim\left\langle\left((\mathbf{Q})^{3} \phi^{(2,+)}\right)(x)\left[\mathbf{Q}^{4}, \phi_{I}^{\left(\mathcal{R}_{1}, \vec{m}_{1}\right)}\right]\left(x_{1}\right) \phi_{J}^{\left(\mathcal{R}_{2},+\right)}\left(x_{2}\right) \phi_{K}^{\left(\mathcal{R}_{3},-\right)}\left(x_{3}\right)\right\rangle
$$

The null condition applied to the operator at $x_{1}$ will generically give supercharges $\mathbf{Q}^{i}$ with $i=1,2,3$, therefore if we want to use a Ward identity to argue that $\mathcal{I}$ vanishes, the operator at $x_{2}$ and $x_{3}$ should be $1 / 2$ BPS operators. ${ }^{17}$ As a consequence, the proof seems to work only for the case $1 / 8 \otimes 1 / 2 \otimes 1 / 2$.

A simple application of the Berenstein-Zelevinsky triangles shows that a product of the form $[p, q, r] \otimes\left[0, k_{1}, 0\right] \otimes\left[0, k_{2}, 0\right]$ contains the trivial representation only if $p=r$, which implies that the the operator at $x_{1}$ must be $1 / 4$ BPS. In this case, if the trivial representation does appear, it appears only one time and the relative Clebsh-Gordan coefficient is unique. Furthermore, since the highest-weight of a $1 / 4$ BPS operator is also annihilated by a right chiral supercharge $\overline{\mathbf{Q}}_{4}$, the proof works for the $\overline{\mathcal{O}}_{\tau}$ operator as well.

Summarizing, we were able to generalize the non-renormalization proof to the 3-point function of one 1/4 BPS operator and two $1 / 2$ BPS operators, but the proof seems to fail in more general cases.

\section{Discussions}

We proved the non-renormalization of certain correlation functions of chiral primary operators in $4 \mathrm{~d} \mathcal{N}=4$ and $2 \mathrm{~d} \mathcal{N}=(4,4)$ superconformal field theories. Our proof was based on the superconformal Ward identities and not on superspace arguments. While equivalent to the latter, we find that the direct proof offers some conceptual advantages.

It would be interesting to explore further more general correlators, for example three point functions of $1 / 4$ BPS operators, and see whether an argument for their nonrenormalization can be found. Or, alternatively, to identify specific examples of such correlators whose weak and strong coupling values differ.

\footnotetext{
${ }^{16}$ Remember that the supercharges $\mathbf{Q}^{i}$ are spinors, so they also carry a Lorentz index.

${ }^{17}$ If the highest-weight is annihilated by $\mathbf{Q}^{i}$ with $i=1,2$, a simple argument based on unitarity bounds [28] shows that it must be annihilated by $\overline{\mathbf{Q}}_{i}$ with $i=3,4$ as well.
} 
In our paper we have not addressed an interesting phenomenon: under continuous deformations of conformal field theories it is possible for short multiplets to combine into long ones and to lift from the BPS bound. By requiring that the spectrum of operators varies continuously, one can derive certain "selection rules" for the types of states which can combine. These rules can be derived by studying how the characters of long representations of the superconformal algebra split into sums of characters of various other representations, when the former hit the unitarity bound. More formally these rules can be encoded in the statement that the "superconformal index" $[29,30]$ of the theory is invariant under continuous deformations. However, we have some additional information: the deformation of the theory is generated by a marginal operator, which is itself a descendant of a chiral primary. It would be interesting to explore whether this imposes any additional constraints on the possible combinations of short multiplets into long ones, besides those imposed by the superconformal index. We hope to revisit this question in future work.

\section{Acknowledgments}

We would like to thank B. Chowdhury, J. Drummond, M. Guica, N. Lambert, S. Minwalla, S. Raju, S. Ramgoolam for useful discussions. We would like to thank K. Intriligator for useful comments on the draft and especially A. Basu for correspondence.

\section{A Roots and weights}

In this appendix we review some basic facts about Lie algebras in order to set notation. In every finite dimensional Lie algebra $\mathfrak{g}$, characterized by a set of hermitian generators $T_{a}$, there is a maximal subset of commuting generators called Cartan subalgebra, spanned by $H_{i}, i=1, \ldots, m$, where $m$ is called the rank of the algebra.

In a finite-dimensional representation $D$ of the Lie algebra, the generators are represented by matrices; the Cartan generators can be simultaneously diagonalized, i.e. we can find a basis of vectors $|\mu\rangle$ such that

$$
H_{i}|\mu\rangle=\mu_{i}|\mu\rangle
$$

where the weight vectors $\mu$ 's are $m$-component vectors with components $\mu_{i}$. A weight is positive if its last non-zero component is positive and negative if its last non-zero component is negative. ${ }^{18}$ In particular, a weight $\mu^{h}$ such that $\mu^{h}-\mu$ is positive for every weight $\mu$ is called highest weight. If the representation is irreducible, the highest weight is unique.

The Lie algebra is a vector space spanned by its generators $\left|T_{a}\right\rangle$, so we can consider the adjoint representation, defined by the action of the algebra on itself:

$$
T_{a}\left|T_{b}\right\rangle=\left|\left[T_{a}, T_{b}\right]\right\rangle
$$

The basis in which the Cartan subalgebra is diagonal is spanned by $\left\{H_{i}, E_{\alpha}\right\}$, and we have

$$
\left[H_{i}, H_{j}\right]=0, \quad\left[H_{i}, E_{\alpha}\right]=\alpha_{i} E_{\alpha}, \quad\left[E_{\alpha}, E_{-\alpha}\right]=\alpha \cdot H
$$

\footnotetext{
${ }^{18} \mathrm{It}$ is customary to define positive weights as having the first non-zero component positive. Nevertheless, our definition is more convenient for $\mathrm{SU}(N)$ groups.
} 
The weights $\alpha$ of the adjoint representation are called roots. A root is called simple if it is positive and cannot be written as a sum of other positive roots. It is possible to prove that the simple roots are linearly independent and complete, so the number of simple roots is equal to the rank of the algebra $m$. We will label the simple roots by $\alpha^{j}, j=1, \ldots, m$.

Given an irreducible representation $D$ and a weight $\mu$, the state $E_{\alpha}|\mu\rangle$ has weight $\mu^{\prime}=\mu+\alpha$ if $E_{\alpha}|\mu\rangle \neq 0$. We will refer to the $E_{\alpha^{j}}$ as raising operators and $E_{-\alpha^{j}}=E_{\alpha^{j}}^{\dagger}$ as lowering operators. In particular, the highest weight is annihilated by the raising operators:

$$
E_{\alpha^{j}}\left|\mu^{h}\right\rangle=0
$$

since $\mu^{h}+\alpha$ is not a weight if $\alpha$ is positive. It is possible to show that

$$
\frac{2 \alpha^{j} \cdot \mu^{h}}{\alpha^{j} \cdot \alpha^{j}}=\ell^{j}
$$

where the $\ell^{j}$ are non-negative integers called Dynkin coefficients.

It is convenient to introduce a basis of weight vectors $\mu^{j}$ such that

$$
\frac{2 \alpha^{j} \cdot \mu^{k}}{\alpha^{j} \cdot \alpha^{j}}=\delta^{j k}
$$

so that the highest weight can be written as $\mu^{h}=\sum_{j} \ell^{j} \mu^{j}$. The $\mu^{j}$ 's are called fundamental weights. Given the highest weight state, all the states in its irreducible representation can be obtained by acting with lowering operators:

$$
E_{-\alpha^{j_{1}}} E_{-\alpha^{j_{2}}} \cdots E_{-\alpha^{j_{n}}}\left|\mu^{h}\right\rangle
$$

where $\alpha^{j_{k}}$ are simple roots. The procedure stops when a state of zero norm is reached. Therefore an irreducible representation is completely characterized by its highest weight state and can be reconstructed by acting on this state with lowering operators associated to simple roots.

As a simple application, notice that if a state has weight $\mu=\sum_{i} k^{i} \mu^{i}$ with $k^{j}=0$ for a given $j$, it is annihilated by the lowering operator $E_{-\alpha^{j}}$. In fact, we have

$$
\left\langle\mu\left|E_{\alpha^{j}} E_{-\alpha^{j}}\right| \mu\right\rangle=\alpha^{j} \cdot \mu\langle\mu \mid \mu\rangle=0
$$

so that $E_{-\alpha^{1}}|\mu\rangle$ is a zero-norm state.

\section{B $1 / 2$ BPS multiplets in $\mathcal{N}=4$}

A detailed analysis of the short multiplets in $\mathcal{N}=4$ can be found in [28]. Let us start with some group-theoretic elements. The R-symmetry group of the $\mathcal{N}=4$ algebra in 4 dimensions is $\mathrm{SU}(4)$. Its Lie algebra has rank 3, and the Cartan generators are given by:

$$
H_{1}=\frac{1}{2}\left(\begin{array}{cccc}
1 & 0 & 0 & 0 \\
0 & -1 & 0 & 0 \\
0 & 0 & 0 & 0 \\
0 & 0 & 0 & 0
\end{array}\right), H_{2}=\frac{1}{\sqrt{12}}\left(\begin{array}{cccc}
1 & 0 & 0 & 0 \\
0 & 1 & 0 & 0 \\
0 & 0 & -2 & 0 \\
0 & 0 & 0 & 0
\end{array}\right), H_{3}=\frac{1}{\sqrt{24}}\left(\begin{array}{cccc}
1 & 0 & 0 & 0 \\
0 & 1 & 0 & 0 \\
0 & 0 & 1 & 0 \\
0 & 0 & 0 & -3
\end{array}\right)
$$


The weights of the fundamental representation are given by

$$
v^{1}=\left(\begin{array}{c}
\frac{1}{2} \\
\frac{1}{\sqrt{12}} \\
\frac{1}{\sqrt{24}}
\end{array}\right), \quad v^{2}=\left(\begin{array}{c}
-\frac{1}{2} \\
\frac{1}{\sqrt{12}} \\
\frac{1}{\sqrt{24}}
\end{array}\right), \quad v^{3}=\left(\begin{array}{c}
0 \\
-\frac{2}{\sqrt{12}} \\
\frac{1}{\sqrt{24}}
\end{array}\right), \quad v^{4}=\left(\begin{array}{c}
0 \\
0 \\
-\frac{3}{\sqrt{24}}
\end{array}\right)
$$

the roots by

$$
\alpha^{1}=v^{1}-v^{2}=\left(\begin{array}{l}
1 \\
0 \\
0
\end{array}\right), \quad \alpha^{2}=v^{2}-v^{3}=\left(\begin{array}{c}
-\frac{1}{2} \\
\frac{\sqrt{3}}{2} \\
0
\end{array}\right), \quad \alpha^{3}=v^{3}-v^{4}=\left(\begin{array}{c}
0 \\
-\frac{1}{\sqrt{3}} \\
\frac{2}{\sqrt{6}}
\end{array}\right)
$$

and the fundamental weights by

$$
\mu^{1}=v^{1}=\left(\begin{array}{c}
\frac{1}{2} \\
\frac{1}{\sqrt{12}} \\
\frac{1}{\sqrt{24}}
\end{array}\right), \quad \mu^{2}=v^{1}+v^{2}=\left(\begin{array}{c}
0 \\
\frac{1}{\sqrt{3}} \\
\frac{1}{\sqrt{6}}
\end{array}\right), \quad \mu^{3}=v^{1}+v^{2}+v^{3}=\left(\begin{array}{c}
0 \\
0 \\
\frac{\sqrt{3}}{2 \sqrt{2}}
\end{array}\right)
$$

so that $\frac{2 \alpha^{j} \cdot \mu^{k}}{\alpha^{j} \cdot \alpha^{j}}=\delta^{j k}$. Every irreducible representation is uniquely characterized by the Dynkin label $\left[k_{1}, k_{2}, k_{3}\right]$, meaning that the highest weight is $\mu^{h}=k_{1} \mu^{1}+k_{2} \mu^{2}+k_{3} \mu^{3}$. The complex conjugate of the representation $\left[k_{1}, k_{2}, k_{3}\right]$ is $\left[k_{3}, k_{2}, k_{1}\right]$.

We will denote the raising operators $E_{\alpha^{1}}, E_{\alpha^{2}}$ and $E_{\alpha^{3}}$ by $E_{1}, E_{2}$ and $E_{3}$ respectively, and the corresponding lowering operators by $E_{1}^{\dagger}, E_{2}^{\dagger}$ and $E_{3}^{\dagger}$.

The highest weight for the fundamental representation is $v^{1}=\mu^{1}$, therefore the Dynkin label is simply $[1,0,0]$. Sometimes it is convenient to denote representations by their dimension $\mathbf{d}$, so that the fundamental representation $[1,0,0]$ is denoted by 4 and its complex conjugate $[0,0,1]$ by $\overline{\mathbf{4}}$. Finally, the six-dimensional representation $[0,1,0]$, or $\mathbf{6}$, corresponds to the fundamental representation of $\mathrm{SO}(6)$ through the local isomorphism $\mathrm{SO}(6) \approx \mathrm{SU}(4)$.

Representations $\left[k_{1}, k_{2}, k_{3}\right]$ are conveniently represented in terms of Young tableaux with $k_{3}$ columns with 3 boxes, $k_{2}$ columns with 2 boxes and $k_{1}$ columns with 1 box:

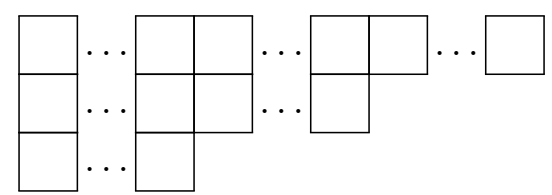

In particular, the fundamental representation $[1,0,0]$ is denoted by

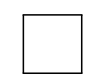

and the representations $[0, k, 0]$ by a Young tableau with $2 k$ boxes:

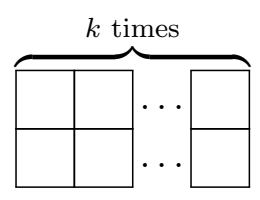

We refer to [31] for more details. 


\section{B.1 The $[0, k, 0]$ multiplet}

The representations of the form $[0, k, 0]$ are of particular importance, since the $1 / 2$ BPS multiplets $\phi^{(k, \vec{m})}$ in the $\mathcal{N}=4$ theory sit in such representations. The vector $\vec{m}$ denotes the weight associated to a particular state in the representation. The highest and lowest weight states are denoted respectively by $\phi^{(k,+)}$ and $\phi^{(k,-)}$.

These representations can be constructed by taking tensor products of $k[0,1,0]$ representations. The $[0,1,0]$, or $\mathbf{6}$, representation can be obtained as the antisymmetric product of two 4 representations. It is usually more convenient to work with a $\mathrm{SO}(6)$ notation $\phi^{i}, i=1, \ldots, 6$. The six scalar fields of $\mathcal{N}=4$ super Yang-Mills sit in this representation. The irreducible representations $[0, k, 0]$ for the chiral primaries correspond to traceless symmetric tensors $C_{i_{1} \ldots i_{k}}$ :

$$
C_{i_{1} \ldots i_{n}} \operatorname{Tr}\left(\phi^{i_{1}} \cdots \phi^{i_{k}}\right)
$$

where the trace is over the $\mathrm{SU}(N)$ gauge group. The highest weight state in this notation is

$$
\operatorname{Tr}\left(Z^{k}\right)=\operatorname{Tr}\left(\left(\phi^{1}+i \phi^{2}\right)^{k}\right)
$$

while the lowest weight is

$$
\operatorname{Tr}\left(\bar{Z}^{k}\right)=\operatorname{Tr}\left(\left(\phi^{1}-i \phi^{2}\right)^{k}\right)
$$

The left-chiral supercharges $\mathbf{Q}$ sit in the fundamental representation of SU(4), and we will use a basis $\mathbf{Q}^{i}, i=1, \ldots, 4$ corresponding to the weights $v^{i}, i=1, \ldots, 4$ defined in equation (B.2) (in this section we ignore the Lorentz indices).

When we act with $\mathbf{Q}$ on $\phi$ we obtain a tensor product representation that can be decomposed as the sum of two irreducible representations as follows

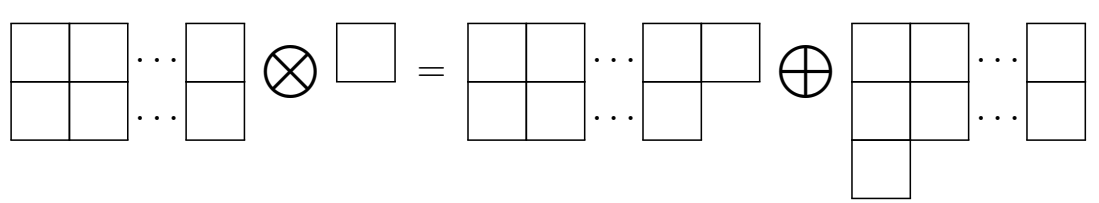

or

$$
[1,0,0] \otimes[0, k, 0]=[1, k, 0] \oplus[0, k-1,1]
$$

Using the $\mathcal{N}=4$ algebra and the condition $\Delta=k$, it is easy to see that the highest weight in $[1, k, 0]$, namely $\left[\mathbf{Q}^{1}, \phi^{(k,+)}\right]$, has zero norm. Furthermore, from equation (A.8) we have $\left[E_{1}^{\dagger}, \phi^{(k,+)}\right]=0$, which means that:

$$
\left[E_{1}^{\dagger},\left[\mathbf{Q}^{1}, \phi^{(k,+)}\right]\right]=\left[\left[E_{1}^{\dagger}, \mathbf{Q}^{1}\right], \phi^{(k,+)}\right]=\left[\mathbf{Q}^{2}, \phi^{(k,+)}\right]
$$

Therefore $\left[\mathbf{Q}^{2}, \phi^{(k,+)}\right]$ belongs to the null representation as well, being a descendant of the highest weight $\left[\mathbf{Q}^{1}, \phi^{(k,+)}\right]$. Therefore we will write

$$
\left[\mathbf{Q}^{1}, \phi^{(k,+)}\right]=0, \quad\left[\mathbf{Q}^{2}, \phi^{(k,+)}\right]=0
$$

Analogously, we have

$$
\left[\mathbf{Q}^{3}, \phi^{(k,-)}\right]=0, \quad\left[\mathbf{Q}^{4}, \phi^{(k,-)}\right]=0
$$


Finally, notice that the decomposition of $\left[k_{1}, k_{2}, k_{3}\right] \otimes\left[k_{1}^{\prime}, k_{2}^{\prime}, k_{3}^{\prime}\right]$ into a sum of irreducible representations contains the trivial representation if and only if $\left[k_{1}^{\prime}, k_{2}^{\prime}, k_{3}^{\prime}\right]$ is the complex conjugate representation of $\left[k_{1}, k_{2}, k_{3}\right]$, that is $\left[k_{3}, k_{2}, k_{1}\right]$. In particular, the tensor product $[0, k, 0] \otimes R$, where $R$ is an arbitrary (not necessarily irreducible) representation, contains the trivial representation if and only if $R$ contains the representation $[0, k, 0]$.

\section{B.2 The $[0,2,0]$ multiplet}

We summarize some (well known) facts about the $[0,2,0] 1 / 2$ BPS multiplet of $\mathcal{N}=4$ SYM. This multiplet is special because it contains the conserved currents and also the marginal operators.

The highest weight of the multiplet is the operator $\operatorname{Tr}\left(Z^{2}\right)$, where $Z=\phi^{1}+i \phi^{2}$. This operator is annihilated by $1 / 2$ of the left chiral and $1 / 2$ of the right chiral supercharges. Here we use the notation $\mathbf{Q}_{a}^{i}, \overline{\mathbf{Q}}_{j, \dot{a}}$ where $i, j$ are $\mathrm{SU}(4)$ indices and $a, \dot{a}$ are $(1 / 2,0)$ and $(0,1 / 2)$ Lorentz spinor indices. The operator $\operatorname{Tr}\left(Z^{2}\right)$ is annihilated by the left chiral $\mathbf{Q}_{a}^{1}, \mathbf{Q}_{a}^{2}$ and the right chiral $\overline{\mathbf{Q}}_{3, \dot{a}}, \overline{\mathbf{Q}}_{4, \dot{a}}$, and is not annihilated by the rest of the supercharges.

Let us consider the four left chiral supercharges which do not annihilate the operator $\operatorname{Tr}\left(Z^{2}\right)$, namely $\mathbf{Q}_{a}^{3}, \mathbf{Q}_{a}^{4}$ where the spinor indices can be $a=1,2$. We notice that according to the $\mathcal{N}=4$ superconformal algebra, these operators anticommute among themselves. Hence if we consider a nested (anti)-commutator of these supercharges, then the order in which the supercharges appear is not important and we can bring them to any desired order. The marginal operator $\mathcal{O}_{\tau}$ can then be written as

$$
\mathcal{O}_{\tau}=\left\{\mathbf{Q}_{1}^{4},\left[\mathbf{Q}_{2}^{4},\left\{\mathbf{Q}_{1}^{3},\left[\mathbf{Q}_{2}^{3}, \operatorname{Tr}\left(Z^{2}\right)\right]\right\}\right]\right\}
$$

It is straightforward to check using the superconformal algebra that this operator is Lorentz scalar, conformal primary and has $\Delta=4$. Similarly, if we act on it with the four right chiral supercharges which do not annihilate it we get the conjugate marginal opeator

$$
\overline{\mathcal{O}}_{\tau}=\left\{\overline{\mathbf{Q}}_{2, \dot{1}},\left[\overline{\mathbf{Q}}_{2, \dot{2}},\left\{\overline{\mathbf{Q}}_{1, \dot{1}},\left[\overline{\mathbf{Q}}_{1, \dot{2}}, \operatorname{Tr}\left(Z^{2}\right)\right]\right\}\right]\right\}
$$

Similar statements hold for the conjugate operator $\operatorname{Tr}\left(\bar{Z}^{2}\right)$, which is the $\mathrm{SU}(4)$ lowest weight state of the $[0,2,0]$ multiplet. This operator is also annihilated by $1 / 2$ of the left chiral and $1 / 2$ of the right chiral supercharges - more specifically it is annihilated by $\mathbf{Q}_{a}^{3}, \mathbf{Q}_{a}^{4}$ and $\overline{\mathbf{Q}}_{1, \dot{a}}, \overline{\mathbf{Q}}_{2, \dot{a}}$. If we act on it with the four left chiral supercharges which do not annihilate it we have

$$
\mathcal{O}_{\tau}=\left\{\mathbf{Q}_{1}^{2},\left[\mathbf{Q}_{2}^{2},\left\{\mathbf{Q}_{1}^{1},\left[\mathbf{Q}_{2}^{1}, \operatorname{Tr}\left(\bar{Z}^{2}\right)\right]\right\}\right]\right\}
$$

while acting with the right chiral supercharges

$$
\overline{\mathcal{O}}_{\tau}=\left\{\overline{\mathbf{Q}}_{4, \dot{1}},\left[\overline{\mathbf{Q}}_{4, \dot{2}},\left\{\overline{\mathbf{Q}}_{3, \dot{1}},\left[\overline{\mathbf{Q}}_{3, \dot{2}}, \operatorname{Tr}\left(\bar{Z}^{2}\right)\right]\right\}\right]\right\}
$$

The expressions (B.16) and (B.19) are manifestly related by complex conjugation. On the other hand, the fact that $\mathcal{O}_{\tau}$ (and similarly $\overline{\mathcal{O}}_{\tau}$ ) can be written either as (B.16) or (B.18) is less obvious and special to $\mathcal{N}=4$ theories. 
The reason that we went into such a detailed presentation here is because the marginal operators in the $\mathcal{N}=4$ have some special properties, which differ from those encountered in theories with less supersymmetry. If we think of the operator $\operatorname{Tr}\left(Z^{2}\right)$ as a "chiral primary" and that of $\operatorname{Tr}\left(\bar{Z}^{2}\right)$ as an "anti-chiral", we notice that both the holomorphic $\mathcal{O}_{\tau}$ and antiholomorphic $\overline{\mathcal{O}}_{\tau}$ marginal operators can be written as descendant of either the chiral or the anti-chiral primary. This is in contrast to what happens in less supersymmetric theories, where the holomorphic deformations are paired with descendants of chiral primaries and anti-holomorphic with descendants of anti-chiral.

Similar special properties of marginal operators are encountered in $2 \mathrm{~d} \mathcal{N}=(4,4)$ theories, as explained in detail in [18].

\section{Null states and short multiplets}

In this appendix we prove the null conditions (4.1) and (5.4). The proof is very similar in both cases, and we begin with the two-dimensional case which is technically simpler.

\section{C.1 Structure of null conditions in $\mathcal{N}=(4,4)$}

For simplicity we drop all extra indices/boldface notation and denote the supercharges by $G^{ \pm} \equiv \mathbf{G}_{-\frac{1}{2}}^{ \pm r}, J \equiv J^{-}$and $\phi=\phi^{(j, j)}$, i.e. the highest weight state in the (short) representation. Also for simplicity we assume that the highest weight state is bosonic (if fermionic some commutators have to be replaced by anticommutators). By definition we have $\left[G^{+}, \phi\right]=0$. What we want to prove is that

$$
\left.[G^{+}, \overbrace{[J, \ldots[J,}^{n}, \phi] \ldots\right] \sim[G^{-}, \overbrace{[J, \ldots[J, \phi]}^{n-1}, \ldots]
$$

We will prove it recursively. For $n=1$ we have

$$
\left[G^{+},[J, \phi]\right]=\left[\left[G^{+}, J\right], \phi\right]+\left[J,\left[G^{+}, \phi\right]\right]=\left[G^{-}, \phi\right]
$$

where we used that the second term is zero and the algebra relation $\left[G^{+}, J\right]=G^{-}$.

Next, let us assume that the condition is true for $n$ and show that it also true for $n+1$. We have

$$
\begin{aligned}
{\left.[G^{+}, \overbrace{[J, \ldots[J}^{n+1}, \phi] \ldots\right] } & \left.=[\left[G^{+}, J\right], \overbrace{[J, \ldots[J}^{n}, \phi] \ldots\right]+\left[J,[G^{+}, \overbrace{[J, \ldots[J}^{n}, \phi] \ldots\right] \\
& \left.=[G^{-}, \overbrace{[J, \ldots[J}^{n}, \phi] \ldots\right]+\left[J,[G^{-}, \overbrace{[J, \ldots[J}^{n-1} \phi] \ldots\right]
\end{aligned}
$$

To get this we used the algebra $\left[G^{+}, J\right]=G^{-}$and the inductive hypothesis. Now we commute $G^{-}$to the left and we have

$$
\left.[G^{+}, \overbrace{[J, \ldots[J}^{n+1}, \phi] \ldots\right]=[G^{-}, \overbrace{[J, \ldots[J, \phi]}^{n} \ldots]+[\left[J, G^{-}\right], \overbrace{[J, \ldots[J, \phi]}^{n-1} \ldots]
$$

Now from the algebra we have $\left[J, G^{-}\right]=0$, so we have proved the desired relation. 


\section{C.2 Structure of null conditions for $\mathcal{N}=4$}

We now move to the four dimensional case where we want to prove (5.4), which reads

$$
\left[\mathbf{Q}^{\star}, \phi_{I}^{\left(k_{1}, \vec{m}_{1}\right)}\right]=\sum_{j \neq \star}\left[\mathbf{Q}^{j}, \mathcal{X}_{j}\right]
$$

here all supercharges are left chiral. We have chosen a basis of supercharges that have definite weight under the Cartan subalgebra. Let us consider one of the supercharges that annihilate a highest weight state $\phi^{(k,+)}$ (namely either $\mathbf{Q}^{1}$ or $\mathbf{Q}^{2}$ ) and call it $\mathbf{Q}^{\star}$. Hence we have

$$
\left[\mathbf{Q}^{\star}, \phi^{(k,+)}\right]=0
$$

In this case equation (C.1) is trivially satisfied.

Let us prove equation (C.1) in the case where the operator is the first $\mathrm{SU}(4)$ "descendant" i.e. $\left[E_{i}^{\dagger}, \phi^{(k,+)}\right]$. We have

$$
\left[\mathbf{Q}^{\star},\left[E_{i}^{\dagger}, \phi^{(k,+)}\right]\right]=\left[E_{i}^{\dagger},\left[\mathbf{Q}^{\star}, \phi^{(k,+)}\right]\right]+\left[\left[\mathbf{Q}^{\star}, E_{i}^{\dagger}\right], \phi^{(k,+)}\right]=\left[\mathbf{Q}^{\prime}, \phi^{(k,+)}\right]
$$

The first term is zero while the term $\left[\mathbf{Q}^{\star}, E_{i}^{\dagger}\right]=\mathbf{Q}^{\prime}$ is another supercharge. However the important point is that the $\mathrm{SU}(4)$ weight of the supercharge $\mathbf{Q}^{\prime}$ is equal to the weight of $\mathbf{Q}^{*}$ minus the root $\alpha_{i}$, so definitely $\mathbf{Q}^{\prime} \neq \mathbf{Q}^{\star}$. Hence (C.1) is proven in this case.

In general, let us assume that the relation is true for an $n$ descendant, that is

$$
\left[\mathbf{Q}^{\star},\left[E_{i_{1}}^{\dagger},\left[\ldots,\left[E_{i_{n}}^{\dagger}, \phi^{(k,+)}\right] \ldots\right]=\sum_{i \neq \star}\left[\mathbf{Q}^{i}, \phi^{\left(k, \vec{m}_{i}\right)}\right]\right.\right.
$$

where the weight of each $\mathbf{Q}^{i}$ is strictly smaller than that of $\mathbf{Q}^{\star}$. We now show that the relation holds for an $n+1$ descendant as well. We have

$$
\begin{aligned}
{\left[\mathbf{Q}^{\star},\left[E_{i}^{\dagger},\left[E_{i_{1}}^{\dagger}, \ldots\left[E_{i_{n}}^{\dagger}, \phi^{(k,+)}\right] \ldots\right]=\left[E_{i}^{\dagger},\right.\right.\right.} & {\left[\mathbf{Q}^{\star},\left[E_{i_{1}}^{\dagger}, \ldots\left[E_{i_{n}}^{\dagger}, \phi^{(k,+)}\right] \ldots\right]\right.} \\
& +\left[\left[\mathbf{Q}^{\star}, E_{i}^{\dagger}\right],\left[E_{i_{1}}^{\dagger}, \ldots\left[E_{i_{n}}^{\dagger}, \phi^{(k,+)}\right] \ldots\right]\right.
\end{aligned}
$$

By using the inductive hypothesis (C.2) on the right hand side, we have

$$
\left[\mathbf{Q}^{\star},\left[E_{i}^{\dagger},\left[E_{i_{1}}^{\dagger} \ldots,\left[E_{i_{n}}^{\dagger}, \phi^{(k,+)}\right] \ldots\right]=\left[E_{i}^{\dagger}, \sum_{j \neq \star}\left[\mathbf{Q}^{j}, \phi^{\left(k, \vec{m}_{j}\right)}\right]\right]+\left[\mathbf{Q}^{\prime},\left[E_{i_{1}}^{\dagger} \ldots\left[E_{i_{n}}^{\dagger}, \phi^{(k,+)}\right] \ldots\right]\right.\right.\right.
$$

where the weight of $\mathbf{Q}^{\prime} \equiv\left[\mathbf{Q}^{\star}, E_{i}^{\dagger}\right]$ is strictly smaller than the weight of $\mathbf{Q}^{\star}$. A further manipulation gives

$$
\begin{aligned}
{\left[\mathbf{Q}^{\star},\left[E_{i}^{\dagger},\left[E_{i_{1}}^{\dagger} \ldots\left[E_{i_{n}}^{\dagger}, \phi^{(k,+)}\right] \ldots\right]=\right.\right.} & \sum_{j \neq \star}\left[\mathbf{Q}^{j},\left[E_{i}^{\dagger}, \phi^{\left(k, \vec{m}_{j}\right)}\right]\right] \\
& +\sum_{j \neq \star}\left[\left[E_{i}^{\dagger}, \mathbf{Q}^{j}\right], \phi^{\left(k, \vec{m}_{j}\right)}\right]+\left[\mathbf{Q}^{\prime},\left[E_{i_{1}}^{\dagger} \ldots\left[E_{i_{n}}^{\dagger}, \phi^{(k,+)}\right] \ldots\right]\right.
\end{aligned}
$$

and since $\mathbf{Q}^{\prime \prime} \equiv\left[E_{i}^{\dagger}, \mathbf{Q}^{j}\right]$ has a smaller weight than $\mathbf{Q}^{j}$, we have proved the desired relation. It is trivial to repeat the above steps for $\mathbf{Q}^{3}$ and $\mathbf{Q}^{4}$ by starting with the lowest weight $\phi^{(k,-)}$ and working "upwards". 
Open Access. This article is distributed under the terms of the Creative Commons Attribution License which permits any use, distribution and reproduction in any medium, provided the original author(s) and source are credited.

\section{References}

[1] J.M. Maldacena, The large- $N$ limit of superconformal field theories and supergravity, Int. J. Theor. Phys. 38 (1999) 1113 [Adv. Theor. Math. Phys. 2 (1998) 231] [hep-th/9711200] [INSPIRE].

[2] S. Gubser, I.R. Klebanov and A.M. Polyakov, Gauge theory correlators from noncritical string theory, Phys. Lett. B 428 (1998) 105 [hep-th/9802109] [INSPIRE].

[3] E. Witten, Anti-de Sitter space and holography, Adv. Theor. Math. Phys. 2 (1998) 253 [hep-th/9802150] [INSPIRE].

[4] S. Lee, S. Minwalla, M. Rangamani and N. Seiberg, Three point functions of chiral operators in $D=4, N=4$ SYM at large- $N$, Adv. Theor. Math. Phys. 2 (1998) 697 [hep-th/9806074] [INSPIRE].

[5] E. D'Hoker, D.Z. Freedman and W. Skiba, Field theory tests for correlators in the AdS/CFT correspondence, Phys. Rev. D 59 (1999) 045008 [hep-th/9807098] [INSPIRE].

[6] E. D'Hoker, D.Z. Freedman, S.D. Mathur, A. Matusis and L. Rastelli, Extremal correlators in the AdS/CFT correspondence, hep-th/9908160 [INSPIRE].

[7] M.R. Gaberdiel and I. Kirsch, Worldsheet correlators in $A d S_{3} / C F T_{2}$, JHEP 04 (2007) 050 [hep-th/0703001] [INSPIRE].

[8] A. Dabholkar and A. Pakman, Exact chiral ring of $A d S_{3} / C F T_{2}$, Adv. Theor. Math. Phys. 13 (2009) 409 [hep-th/0703022] [INSPIRE].

[9] A. Pakman and A. Sever, Exact $N=4$ correlators of $A d S_{3} / C F T_{2}$, Phys. Lett. B 652 (2007) 60 [arXiv:0704.3040] [InSPIRE].

[10] M. Taylor, Matching of correlators in $A d S_{3} / C F T_{2}$, JHEP 06 (2008) 010 [arXiv:0709.1838] [INSPIRE].

[11] K.A. Intriligator, Bonus symmetries of $N=4$ super Yang-Mills correlation functions via AdS duality, Nucl. Phys. B 551 (1999) 575 [hep-th/9811047] [INSPIRE].

[12] K.A. Intriligator and W. Skiba, Bonus symmetry and the operator product expansion of $N=4$ Super Yang-Mills, Nucl. Phys. B 559 (1999) 165 [hep-th/9905020] [INSPIRE].

[13] B. Eden, P.S. Howe and P.C. West, Nilpotent invariants in $N=4 S Y M$, Phys. Lett. B 463 (1999) 19 [hep-th/9905085] [INSPIRE].

[14] A. Petkou and K. Skenderis, A nonrenormalization theorem for conformal anomalies, Nucl. Phys. B 561 (1999) 100 [hep-th/9906030] [INSPIRE].

[15] P.S. Howe, C. Schubert, E. Sokatchev and P.C. West, Explicit construction of nilpotent covariants in $N=4$ SYM, Nucl. Phys. B 571 (2000) 71 [hep-th/9910011] [InSPIRE].

[16] P. Heslop and P.S. Howe, OPEs and three-point correlators of protected operators in $N=4$ SYM, Nucl. Phys. B 626 (2002) 265 [hep-th/0107212] [INSPIRE].

[17] A. Basu, M.B. Green and S. Sethi, Some systematics of the coupling constant dependence of $N=4$ Yang-Mills, JHEP 09 (2004) 045 [hep-th/0406231] [INSPIRE]. 
[18] J. de Boer, J. Manschot, K. Papadodimas and E. Verlinde, The chiral ring of $A d S_{3} / C F T_{2}$ and the attractor mechanism, JHEP 03 (2009) 030 [arXiv:0809.0507] [INSPIRE].

[19] K. Ranganathan, H. Sonoda and B. Zwiebach, Connections on the state space over conformal field theories, Nucl. Phys. B 414 (1994) 405 [hep-th/9304053] [INSPIRE].

[20] K. Papadodimas, Topological anti-topological fusion in four-dimensional superconformal field theories, JHEP 08 (2010) 118 [arXiv:0910.4963] [INSPIRE].

[21] N. Seiberg, Observations on the moduli space of superconformal field theories, Nucl. Phys. B 303 (1988) 286 [INSPIRE].

[22] D. Kutasov, Geometry on the space of conformal field theories and contact terms, Phys. Lett. B 220 (1989) 153 [INSPIRE].

[23] C.A. Cardona and I. Kirsch, Worldsheet four-point functions in $A d S_{3} / C F T_{2}$, JHEP 01 (2011) 015 [arXiv: 1007.2720] [INSPIRE]

[24] I. Kirsch and T. Wirtz, Worldsheet operator product expansions and p-point functions in $A d S_{3} / C F T_{2}$, JHEP 10 (2011) 049 [arXiv:1106.5876] [INSPIRE].

[25] A. Strominger and C. Vafa, Microscopic origin of the Bekenstein-Hawking entropy, Phys. Lett. B 379 (1996) 99 [hep-th/9601029] [INSPIRE].

[26] J.M. Maldacena, A. Strominger and E. Witten, Black hole entropy in M-theory, JHEP 12 (1997) 002 [hep-th/9711053] [INSPIRE].

[27] R. Minasian, G.W. Moore and D. Tsimpis, Calabi-Yau black holes and $(0,4) \sigma$-models, Commun. Math. Phys. 209 (2000) 325 [hep-th/9904217] [InSPIRE].

[28] F. Dolan and H. Osborn, On short and semi-short representations for four-dimensional superconformal symmetry, Annals Phys. 307 (2003) 41 [hep-th/0209056] [INSPIRE].

[29] J. Kinney, J.M. Maldacena, S. Minwalla and S. Raju, An index for 4 dimensional super conformal theories, Commun. Math. Phys. 275 (2007) 209 [hep-th/0510251] [INSPIRE].

[30] C. Romelsberger, Counting chiral primaries in $N=1, D=4$ superconformal field theories, Nucl. Phys. B 747 (2006) 329 [hep-th/0510060] [INSPIRE].

[31] H. Georgi, Lie algebras in particle physics. From isospin to unified theories, Front. Phys. $\mathbf{5 4}$ (1982) 1. 